\title{
Continuum model for the long-range elastic interaction on stepped epitaxial surfaces in $2+1$ dimensions
}

\author{
Xiaohong Zhu, Haoyun Xu, and Yang Xiang* \\ Department of Mathematics, Hong Kong University of Science and Technology, Clear Water Bay, Kowloon, Hong Kong
}

(Received 26 September 2008; revised manuscript received 26 December 2008; published 10 March 2009)

\begin{abstract}
In heteroepitaxy, the mismatch of lattice constants between the crystal film and the substrate causes misfit strain and stress in the bulk of the film, driving the surface of the film to self-organize into various nanostructures. Below the roughening transition temperature, an epitaxial surface consists of facets and steps and changes its morphology by lateral motion of steps. In this paper, we present a $2+1$-dimensional continuum model for the long-range elastic interaction on stepped surface of a strained film. The continuum model is derived rigorously from the discrete model for the interaction between steps; thus it incorporates the discrete features of the stepped surfaces. Examples show that our continuum model is much more accurate as an approximation to the discrete model than the traditional continuum approximation. Moreover, in the linear instability of a planar surface, our continuum model gives the transition from step bunching instability to step undulation instability as the distance between adjacent steps increases, which agrees with the experimental observations and the results of discrete models and is missing using the traditional continuum approximation. Numerical simulations of the surface evolution using our model in the nonlinear regime show several different surface morphologies, including the morphology of step bunching which cannot be obtained using the traditional continuum approximation.
\end{abstract}

DOI: 10.1103/PhysRevB.79.125413

PACS number(s): 81.10.Aj, 81.15.-z, 62.20.D-, 68.35.-p

\section{INTRODUCTION}

There has been extensive research on the morphological evolution of surfaces in heteroepitaxial growth, in which the epitaxial film is under stress due to the misfit between the film and the substrate. These stress-driven self-assembled nanostructures exhibit interesting electronic and optical properties and have various potential applications in semiconductor industry. ${ }^{1-4}$

Many continuum models can be found in the literature on the surface morphological evolution under elastic effects, in which the surfaces are modeled as continuously changed profiles without any discrete structures on them., ${ }^{2,5}$ The stress in the solid is a destabilizing factor while the surface energy is a stabilizing one, and the planar surface of a stressed solid is unstable for perturbations with wave numbers less than a critical value. ${ }^{6-10}$ These models work in the regime above the roughening transition temperature. Below the roughening transition temperature, an epitaxial surface consists of facets and steps, which makes the continuum approaches mentioned above not apply directly. In the stepflow model, a stepped surface changes its morphology by lateral motion of steps. ${ }^{1,4,17-19}$ For an unstrained film, the elastic effect of a step can be viewed as a distribution of force dipole on the film surface along the step. The dipole interaction force decays as $1 / r^{3}$ for two parallel straight steps with distance $r$. The elastic effect of a step on the surface of a strained film (in heteroepitaxy) can be approximated by a distribution of force monopole along the step. The force monopole interaction has a long-range effect, which decays as $1 / r$ for two parallel straight steps with distance $r$. Expansions with higher-order terms for the elastic effects of surface steps were obtained in Refs. 20-22. For an epitaxial surface consisting of uniform straight step array, the force monopole effect is destabilizing while the step line energy and force dipole effect are stabilizing. ${ }^{19,23-29}$
Several discrete models have been proposed for elastic interactions and dynamics of straight and parallel steps (1 +1-dimensional models), e.g., by Alerhand et al., ${ }^{19}$ Tersoff and co-workers, ${ }^{24,25}$ Duport et al., ${ }^{26,27}$ Ozdemir and Zangwill, ${ }^{30}$ Kaganer and Ploog. ${ }^{31}$ The elastic interactions cause step bunching instability on strained epitaxial surfaces. Continuum 1+1-dimensional models have also been proposed by Lançon and Villain, ${ }^{32}$ Kaganer and Ploog, ${ }^{31}$ and Shenoy and Freund. ${ }^{33}$ However, for the long-range force monopole interaction on strained epitaxial surfaces, the traditional expression above roughening transition temperature was directly used in these models. ${ }^{31,33}$ Xiang and $E^{34,35}$ derived a continuum model rigorously by taking the continuum limit of the discrete step dynamics models of Tersoff and co-workers ${ }^{24,25}$ and Duport et al. ${ }^{26,27}$ For the long-range force monopole effect, besides the widely used integral expression above the roughening transition temperature, there is another term incorporating the discrete features of the stepped surface in their continuum model. This additional term is crucial in modeling the step bunching instability on stepped surfaces. ${ }^{35}$

In reality, the steps cannot keep straight and tend to meander under many effects including the elastic interactions. 1,4,23,36 The force dipole effect between curved steps on unstrained epitaxial surfaces are relatively well modeled in the frameworks of both the discrete step dynamics $^{37,38}$ and the continuum model. ${ }^{32,37,39-42}$ In the framework of the continuum theory, it is well known that the elastic effect on such a stepped surface of unstrained film is quite different from that on an epitaxial surface above roughening transition temperature due to the discrete structures on the stepped surface.

Only a few 2+1-dimensional models available in the literature account for the long-range force monopole interaction on stepped surfaces, mostly based on discrete step inter- 
action or dynamics. Tersoff and Pehlke ${ }^{23}$ analyzed step undulation instability of stepped $\mathrm{Si}(001)$ surface which is subject to force monopole effect at the steps and their results agree well with the experimental observations obtained by Tromp and Reuter. ${ }^{36}$ Houchmandzadeh and Misbah ${ }^{28}$ studied the force dipole and force monopole elastic interactions between slightly modulated steps. Kukta and Bhattacharya ${ }^{43}$ proposed a $2+1$ step-flow model that accounts for both the elastic effects and terrace diffusion. Léonard and Tersoff ${ }^{29}$ compared the step bunching and step meandering instabilities of a stepped surface under stress for both permeable and impermeable steps. Shenoy ${ }^{44}$ studied the growth of epitaxial nanowires by controlled coarsening of strained islands. Haußer et al. ${ }^{45}$ proposed a step-flow model for the heteroepitaxial growth of strained, substitutional, and binary alloy films with phase segregation. Kaganer and Ploog ${ }^{31}$ investigated the energetics of strained axially symmetric coneshaped stepped surfaces using both approaches of discrete step dynamics and continuum equation. However, their continuum model for the long-range elastic effect on strained stepped surface was obtained as a simple average of the force monopole interaction between steps, which is the same as the traditional integral expression above roughening transition temperature. Ramasubramaniam and Shenoy ${ }^{46}$ generalized the continuum model in $1+1$ dimensions based on continuum variational principles proposed by Shenoy and Freund $^{33}$ to $2+1$ dimensions. Even though the discrete features of the step line energy and force dipole interaction between steps were included in their models, for the long-range elastic interaction due to misfit, the traditional expression above roughening transition temperature was directly used. A continuum theory that accounts for the long-range elastic effect and the discrete features for a strained epitaxial film with a general stepped surface in $2+1$ dimensions, as the continuum equation proposed in Refs. 34 and 35 for a surface with straight steps, is still lacking.

In this paper, we present a 2+1-dimensional continuum model for the long-range elastic interaction on stepped surface of a strained film. The continuum model is derived rigorously from the discrete model for the interaction between steps. As in the $1+1$ continuum equation obtained in Refs. 34 and 35 , the continuum model includes additional terms accounting for the discrete features of the stepped surfaces besides the traditional integral expression above roughening transition temperature. We validate our model by comparing the elastic interaction and linear instability of a planar surface with the results of the discrete model. Examples show that our continuum model is much more accurate as an approximation to the discrete model than the traditional continuum approximation. Moreover, in the linear instability of a planar surface, our continuum model gives the transition from step bunching instability to step undulation instability as the distance between adjacent steps increases, which agrees with the experimental observations and the results of discrete models and is missing using the traditional continuum approximation. Numerical simulations of the surface evolution using our model in the nonlinear regime show several different surface morphologies, including the morphology of step bunching which cannot be obtained using the traditional continuum approximation. Note that rigorous derivation of our model can be found in a previous paper. ${ }^{47} \mathrm{~A}$ simplified derivation for an axisymmetric conical-stepped surface is given in this paper (see the Appendix).

The surface instabilities caused by the above-mentioned elastic effects are energetic instabilities, which are different from the kinetic instabilities during growth caused by, e.g., the step edge barriers. ${ }^{1,48-50}$ Extensive research can be found in the literature on the kinetic instability. The step edge barriers also modify the mobility for the surface evolution under elastic effects. The kinetic instability and the effect of the step edge barriers on the mobility were both incorporated in the 1+1-dimensional continuum model derived from the discrete step dynamic models. ${ }^{34}$ The anisotropic mobility due to step edge barriers in $2+1$ dimensions was obtained by Margetis and Kohn. ${ }^{42}$ The kinetic instabilities and step edge barriers are neglected in this paper and will be considered in the future work.

The rest of this paper is organized as follows. In Sec. II, we briefly review the long-range elastic effect on stepped heteroepitaxial surfaces and the available continuum models. In Sec. III, we present our continuum model for the longrange elastic interaction on stepped epitaxial surfaces. In Sec. IV, we examine the accuracy of our model by an example of comparison with the discrete model. In Sec. V, we study the linear instability of a planar surface to small perturbations. In Sec. VI, we present some simulations of evolution of the stepped surfaces under elastic effects in the nonlinear regime. In Sec. VII, we summarize the results.

\section{LONG-RANGE ELASTIC EFFECT ON STEPPED HETEROEPITAXIAL SURFACES AND AVAILABLE CONTINUUM MODELS}

In this section, we briefly review the long-range elastic effect on stepped heteroepitaxial surfaces and available continuum models for it. More details can be found in the books and reviews ${ }^{1-4}$ and other references in this section.

In heteroepitaxial growth, the misfit of the lattice constants is defined by

$$
\varepsilon_{0}=\frac{a_{f}-a}{a},
$$

where $a_{f}$ and $a$ are the lattice constants of the film and the substrate, respectively. This misfit results in elastic strain and stress fields in the film and the substrate. For an isotropic film with flat surface and infinite substrate, there is a constant stress field in the bulk of the film. When the height of the film is in the $z$ direction, the nonzero components of the stress tensor in the film are

$$
\sigma_{x x}=\sigma_{y y}=\sigma_{0}=\frac{2 G(1+\nu) \varepsilon_{0}}{1-\nu},
$$

where $G$ is the shear modulus, and $\nu$ is the Poisson ratio.

For a heteroepitaxial film with nonflat surface, the elastic state has to be determined by solving elasticity systems in the film and the substrate. For a film with slightly modulated surface subject to the misfit stress, the elasticity system is approximately equivalent to that in a film with flat surface and subject to a traction ${ }^{1-4,6-10}$ 


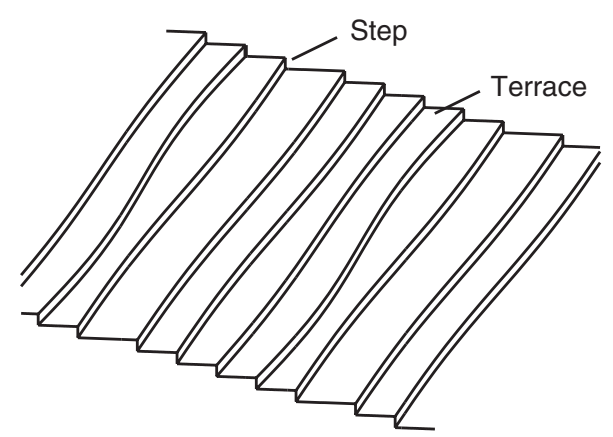

FIG. 1. A stepped epitaxial surface.

$$
\mathbf{T}=-\sigma_{0}\left(h_{x}, h_{y}, 0\right)
$$

on its surface, where $h(x, y)$ is the height of the surface. In this case, the elasticity problem can be solved explicitly using the Green function. ${ }^{51}$

Below the roughening transition temperature, an epitaxial surface consists of atomic-height steps and atomic-flat terraces (see Fig. 1). A step on a heteroepitaxial surface can be considered as a distribution of force monopole along the step with strength $\sigma_{0} a$ in the normal direction of the step $^{1,4,19,23-29,31,33,43,44}$ [see also Eq. (3)]. Using Eq. (3) and the Green function, the interaction energy due to misfit between two steps $\gamma_{1}$ and $\gamma_{2}$ is

$$
\begin{aligned}
E_{\text {misfit }}\left(\gamma_{1}, \gamma_{2}\right)= & \frac{\sigma_{0}^{2} a^{2}}{2 \pi G} \int_{\gamma_{1}} \int_{\gamma_{2}}\left[(1-\nu) \frac{\mathbf{n}_{1} \cdot \mathbf{n}_{2}}{r}\right. \\
& \left.+\nu \frac{\left(\mathbf{n}_{1} \cdot \mathbf{r}\right)\left(\mathbf{n}_{2} \cdot \mathbf{r}\right)}{r^{3}}\right] d l_{1} d l_{2},
\end{aligned}
$$

where $d l_{1}$ and $d l_{2}$ are line elements along $\gamma_{1}$ and $\gamma_{2}$, respectively, $\mathbf{r}$ is the vector between a point on $\gamma_{1}$ and a point on $\gamma_{2}$ with length $r$, and $\mathbf{n}_{1}$ and $\mathbf{n}_{2}$ are the unit normal vectors of $\gamma_{1}$ and $\gamma_{2}$, respectively. The self-misfit energy of a step $\gamma$ is thus $E_{\text {misfit }}(\gamma)=\frac{1}{2} E_{\text {misfit }}(\gamma, \gamma)$.

In some continuum theories, the continuum expressions for strained epitaxial surface above roughening transition temperature were directly used for stepped surfaces, ${ }^{31,33,46}$ in which the surface profile is a smooth function $h(x, y)$ and the traction on the surface is given by Eq. (3). Using this idea, the misfit elastic energy in the continuum framework is given by

$$
\begin{aligned}
E_{\text {misfit }}= & -\frac{(1-\nu) \sigma_{0}^{2}}{4 \pi G} \int_{-\infty}^{\infty} \int_{-\infty}^{\infty} h(x, y) \\
& \times\left[\int_{-\infty}^{\infty} \int_{-\infty}^{\infty} \frac{(x-\xi) h_{x}(\xi, \eta)+(y-\eta) h_{y}(\xi, \eta)}{\left[(x-\xi)^{2}+(y-\eta)^{2}\right]^{3 / 2}} d \xi d \eta\right] \\
& \times d x d y .
\end{aligned}
$$

Xiang and $E^{34,35}$ rigorously derived a continuum model for epitaxial surfaces with straight steps by taking the continuum limit of the discrete models of steps. ${ }^{24-27}$ Their continuum model for the misfit elastic energy on a surface with straight steps, without nucleation of new steps, is

$$
\begin{aligned}
E_{\text {misfit }}= & -\frac{(1-\nu) \sigma_{0}^{2}}{2 \pi G} \int_{-\infty}^{\infty}\left[h(x) \int_{-\infty}^{\infty} \frac{h^{\prime}(\xi)}{x-\xi} d \xi\right. \\
& \left.+a\left|h_{x}(x)\right| \log \left|h_{x}(x)\right|\right] d x .
\end{aligned}
$$

The first term in this expression is the same as the misfit elastic energy above the roughening transition temperature, which is given by Eq. (5) when $h$ is uniform in $y$ direction, and which was used also for stepped surfaces in Ref. 33. The second term in this expression is the correction due to the discrete nature of the stepped surface.

Besides the misfit elastic energy, there are step line energy and the elastic energy due to force dipole interaction for a stepped epitaxial surface. The total energy on a stepped surface due to elastic effects can be written as

$$
E_{\text {total }}=E_{\text {line }}+E_{\text {dipole }}+E_{\text {misfit }},
$$

where, in the continuum framework,

$$
E_{\text {line }}=\int_{-\infty}^{\infty} \int_{-\infty}^{\infty} g_{1}|\nabla h| d x d y
$$

is the step line energy, with $g_{1}$ being the step line energy density, and

$$
E_{\text {dipole }}=\int_{-\infty}^{\infty} \int_{-\infty}^{\infty} \frac{g_{3}}{3}|\nabla h|^{3} d x d y
$$

is the energy due to the force dipole interaction, with $g_{3}$ being the strength of this interaction. The step line energy and the elastic energy due to force dipole interaction are relatively well known in the continuum framework in the literature. ${ }^{1,4,31-35,37,39-42,46}$

A stepped surface changes its morphology by motion, nucleation, and annihilation of steps, instead of changing continuously. Based on the Burton-Cabrera-Frank (BCF) theory, ${ }^{17}$ the evolution equation is given by ${ }^{30,32-35,37,39-42,46}$

$$
\frac{\partial h}{\partial t}=\nabla \cdot(D \nabla \mu),
$$

where $D$ is the mobility depending on the adatom diffusion on the terraces and attachment detachment of adatoms along steps. The total chemical potential $\mu$ on a misfit-strained stepped surface can be written as

$$
\mu=\mu_{s}+\mu_{d}+\mu_{m} .
$$

The first term $\mu_{s}$ is the chemical potential due to the step line energy

$$
\mu_{s}=\frac{\delta E_{\text {line }}}{\delta h}=-\nabla \cdot\left(g_{1} \frac{\nabla h}{|\nabla h|}\right),
$$

and the second term $\mu_{d}$ is the chemical potential due to the interaction between steps in unstrained films which can be approximated by force dipole interaction

$$
\mu_{d}=\frac{\delta E_{\text {dipole }}}{\delta h}=-\nabla \cdot\left(g_{3}|\nabla h| \nabla h\right) .
$$

The last term 


$$
\mu_{m}=\frac{\delta E_{\text {misfit }}}{\delta h}
$$

in the above total chemical-potential expression is the contribution of the long-range elastic interaction due to the misfit.

Using Eq. (5), the chemical potential due to misfit is $\mu_{m}(x, y)=\mu_{m}^{0}(x, y)$, where

$$
\begin{aligned}
\mu_{m}^{0}(x, y)= & -\frac{(1-\nu) \sigma_{0}^{2}}{2 \pi G} \\
& \times \int_{-\infty}^{\infty} \int_{-\infty}^{\infty} \frac{(x-\xi) h_{x}(\xi, \eta)+(y-\eta) h_{y}(\xi, \eta)}{\left[(x-\xi)^{2}+(y-\eta)^{2}\right]^{3 / 2}} d \xi d \eta .
\end{aligned}
$$

However, this expression is the same as that above the roughening transition temperature, and the discrete features of the stepped surface are not included. In the continuum 1 +1-dimensional model of Xiang and E, ${ }^{34,35}$ the chemical potential due to misfit is

$$
\mu_{m}(x)=-\frac{(1-\nu) \sigma_{0}^{2}}{\pi G} \int_{-\infty}^{\infty} \frac{h^{\prime}(\xi)}{x-\xi} d \xi-\frac{(1-\nu) \sigma_{0}^{2} a}{2 \pi G} \frac{h_{x x}(x)}{\left|h_{x}(x)\right|}
$$

The integral term in this expression is the corresponding term of Eq. (15) when $h$ is uniform in the $y$ direction. The second term in this expression incorporates the discrete nature of the stepped surface, which is crucial in modeling the step bunching instability on stepped surfaces. ${ }^{35}$ In this paper, we shall generalize this continuum model to $2+1$ dimensions for epitaxial surfaces with curved steps.

\section{CONTINUUM MODEL}

In this section, we present our 2+1-dimensional continuum model for the long-range elastic effect on stepped surfaces, which is derived from the discrete model for the interaction between steps.

We first describe the discrete model. Consider a stepped surface whose profile is given exactly by $\tilde{h}(x, y)$. Assume that $\left\{\gamma_{j}\right\}, j=\ldots,-2,-1,0,1,2, \ldots$, are steps on the surface. Using the relation

$$
\nabla \tilde{h}=-a \sum_{j} \delta\left(\gamma_{j}\right) \mathbf{n}_{j}
$$

in Eq. (15), where $\delta\left(\gamma_{j}\right)$ is the one-dimensional Dirac delta function in the normal direction $\mathbf{n}_{j}$ of the step $\gamma_{j}$, the chemical potential at a point $\mathbf{X}=(x, y)$ on step $\gamma_{n}$ is

$$
\tilde{\mu}_{m}(x, y)=\tilde{\mu}_{m}^{\text {int }}(x, y)+\tilde{\mu}_{m}^{\text {self }}(x, y),
$$

with

$$
\tilde{\mu}_{m}^{\mathrm{int}}(x, y)=\frac{(1-\nu) \sigma_{0}^{2} a}{2 \pi G}\left(\sum_{j \neq n} \int_{\gamma_{j}} \frac{\left(\mathbf{X}-\mathbf{X}_{1}\right) \cdot \mathbf{n}_{j}\left(\mathbf{X}_{1}\right)}{\left\|\mathbf{X}-\mathbf{X}_{1}\right\|^{3}} d l\right)
$$

and

$$
\begin{aligned}
& \tilde{\mu}_{m}^{\text {self }}(x, y) \\
& \quad=\frac{(1-\nu) \sigma_{0}^{2} a}{2 \pi G}\left(\int_{-\infty}^{\infty} \delta(\omega) d \omega \int_{\gamma_{\omega}} \frac{\left(\mathbf{X}-\mathbf{X}_{1}\right) \cdot \mathbf{n}_{\omega}\left(\mathbf{X}_{1}\right)}{\left\|\mathbf{X}-\mathbf{X}_{1}\right\|^{3}} d l\right),
\end{aligned}
$$

where $\mathbf{X}_{1}$ is a point that varies along the steps in these line integrals, the curve $\gamma_{\omega}=\left\{\mathbf{X}_{0}+\omega \mathbf{n}_{n}\left(\mathbf{X}_{0}\right): \mathbf{X}_{0} \in \gamma_{n}\right\}$, and $\mathbf{n}_{\omega}$ is its unit normal vector. Note that for the two possible directions of the normal direction of a step, we choose it to be the direction in which the surface height is decreasing. The above formulas for chemical potential can also be obtained by taking variation of the self-energy and interaction energy with respect to the position of the step.

The delta function $\delta(\omega)$ in the chemical potential due to the self-interaction $\tilde{\mu}_{m}^{\text {self }}(x, y)$ has to be regularized to avoid nonphysical singularity (same notation is used for simplicity). The width of the regularization of $\delta(\omega)$, which reflects the detailed structure of the step and can be determined from atomistic calculations, is of the order of the lattice constant $a$. The regularization or cutoff is commonly used to remove nonphysical singularities in the models of steps. ${ }^{4,19,29,43,44}$

Although Eqs. (18)-(20) give an exact expression for the misfit-induced long-range elastic interaction on a stepped surface, in the framework of a continuum model, the stepped surface is often described by a smooth profile $h(x, y)$ without resolving the details of the steps. In our continuum model, the surface profile $h(x, y)$ connects the steps smoothly so that a step $\gamma_{n}$ with height $h_{n}$ is the contour line $h(x, y)=h_{n}$ (see Fig. 2).

From the discrete model given by Eqs. (18)-(20), letting the lattice constant $a \rightarrow 0$ in the length unit of the continuum model, which means that a large number of steps are contained in a unit area in the continuum model, we have the continuum model for the long-range elastic interaction on a stepped surface in heteroepitaxy in $2+1$ dimensions

$$
\begin{aligned}
\mu_{m}(x, y)= & -\frac{(1-\nu) \sigma_{0}^{2}}{2 \pi G} \\
& \times \int_{-\infty}^{\infty} \int_{-\infty}^{\infty} \frac{(x-\xi) h_{x}(\xi, \eta)+(y-\eta) h_{y}(\xi, \eta)}{\left[(x-\xi)^{2}+(y-\eta)^{2}\right]^{3 / 2}} d \xi d \eta \\
& -\frac{(1-\nu) \sigma_{0}^{2} a}{2 \pi G}\left[\nabla \cdot\left(\frac{\nabla h}{|\nabla h|}\right) \log \frac{2 \pi r_{c}|\nabla h|}{a}\right. \\
& \left.+\frac{\nabla h D^{2} h \nabla^{T} h}{|\nabla h|^{3}}\right],
\end{aligned}
$$

where $r_{c}$ is a parameter depending on the core of the step

$$
\log r_{c}=\int_{-\infty}^{\infty} \delta(\omega) \log |\omega| d \omega
$$

$D^{2} h$ is the Hessian matrix of $h(x, y)$, and $\nabla h D^{2} h \nabla^{T} h=h_{x}^{2} h_{x x}$ $+2 h_{x} h_{y} h_{x y}+h_{y}^{2} h_{y y}$. In this continuum expression, the term of the integral over the whole surface is the same as the expression of the misfit-induced elastic interaction above the roughening transition temperature, and two additional terms incorporate the atomic feature of the stepped surface. 


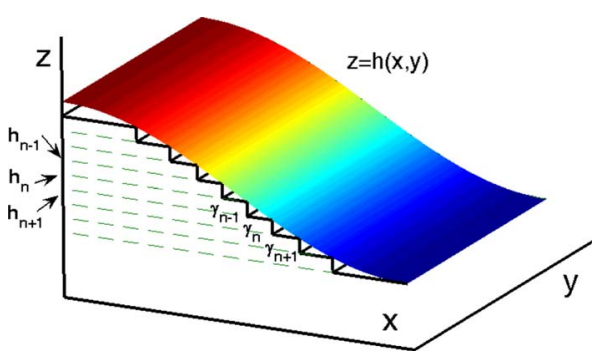

FIG. 2. (Color online) A stepped surface described by a smooth profile $h(x, y)$.

It is easy to verify that this continuum model is reduced to the 1+1-dimensional model for a surface with straight steps given by Eq. (16). ${ }^{34,35}$ For an axisymmetric conicalmoundlike-stepped surface $h(r)$ with $h^{\prime}(r)<0$, where $r$ is the radial coordinate, the result becomes

$$
\begin{aligned}
\mu_{m}(r)= & \frac{(1-\nu) \sigma_{0}^{2}}{\pi G} \int_{0}^{\infty}\left(\frac{K(m)}{\hat{r}+r}+\frac{E(m)}{\hat{r}-r}\right) h^{\prime}(\hat{r}) d \hat{r} \\
& +\frac{(1-\nu) \sigma_{0}^{2} a}{2 \pi G}\left(\frac{1}{r} \log \frac{2 \pi r_{c}\left|h^{\prime}(r)\right|}{a}+\frac{h^{\prime \prime}(r)}{h^{\prime}(r)}\right),
\end{aligned}
$$

where $K(m)=\int_{0}^{\pi / 2} \frac{d \theta}{\sqrt{1-m \sin ^{2} \theta}}$ and $E(m)=\int_{0}^{\pi / 2} \sqrt{1-m \sin ^{2} \theta} d \theta$ are the complete elliptic integrals of the first and second kinds, respectively, with $m=\frac{4 \hat{r} r}{(\hat{r}+r)^{2}}$.

The detailed derivation of Eq. (21) is technical and has been given in a previous paper. ${ }^{47}$ To show the main ideas, we give a simplified derivation for Eq. (23) for an axisymmetric conical-stepped surface in the Appendix.

The derived continuum expression of chemical potential given by Eq. (21) can be written as the variation of an elastic energy

$$
\begin{aligned}
E_{\text {misfit }}= & -\frac{(1-\nu) \sigma_{0}^{2}}{4 \pi G} \int_{-\infty}^{\infty} \int_{-\infty}^{\infty} h(x, y) \\
& \times\left[\int_{-\infty}^{\infty} \int_{-\infty}^{\infty} \frac{(x-\xi) h_{x}(\xi, \eta)+(y-\eta) h_{y}(\xi, \eta)}{\left[(x-\xi)^{2}+(y-\eta)^{2}\right]^{3 / 2}} d \xi d \eta\right] \\
& \times d x d y+\frac{(1-\nu) \sigma_{0}^{2} a}{2 \pi G} \int_{-\infty}^{\infty} \int_{-\infty}^{\infty}|\nabla h| \log \frac{2 \pi r_{c}|\nabla h|}{e a} d x d y,
\end{aligned}
$$

where the constant $e$ is the base of the natural logarithm. The first term in this expression is the traditional expression of the misfit elastic energy above the roughening transition temperature on surfaces with small amplitude modulation. The second term is the additional term we obtained which is due to the contribution to the step line energy from the force monopole interaction and which gives the two local terms in the chemical potential in Eq. (21).

For a surface with straight steps, this continuum model is reduced to the 1+1-dimensional model given by Eq. (6). ${ }^{34,35}$ Note that in the $1+1$ model, the term of $\left|h_{x}\right|$ with constant coefficient in the total energy, i.e., the step line energy with constant density, plays no role in the surface morphology evolution when nucleation is neglected; thus this kind of term does not appear in the $1+1$ model in Eq. (6). The total misfit elastic energy of a surface consisting of a uniform step train has also been calculated using the discrete model, ${ }^{19}$ whose density is

$$
e_{\text {misfit }}=-\frac{(1-\nu) \sigma_{0}^{2} a^{2}}{2 \pi G l_{t}} \log \frac{l_{t}}{\pi r_{0}},
$$

where $l_{t}$ is the distance between two adjacent steps, and $r_{0}$ is a cutoff distance. In this case, using the relation $\left|h^{\prime}(x)\right|$ $=a / l_{t}$, our model (24) gives the misfit elastic energy density

$$
e_{\text {misfit }}=-\frac{(1-\nu) \sigma_{0}^{2} a^{2}}{2 \pi G l_{t}} \log \frac{e l_{t}}{2 \pi r_{c}},
$$

which agrees with the result of the discrete model. (Note that the values of the cutoff distance are different in these two models.)

Finally, using Eqs. (10)-(13) and (21), we have the morphological evolution equation of a stepped surface under elastic effects

$$
\begin{aligned}
\frac{\partial h}{\partial t}= & \nabla \cdot\left\{D \nabla \left[-\nabla \cdot\left(g_{1} \frac{\nabla h}{|\nabla h|}+g_{3}|\nabla h| \nabla h\right)-\frac{(1-\nu) \sigma_{0}^{2}}{2 \pi G}\right.\right. \\
& \times \int_{-\infty}^{\infty} \int_{-\infty}^{\infty} \frac{(x-\xi) h_{x}(\xi, \eta)+(y-\eta) h_{y}(\xi, \eta)}{\left[(x-\xi)^{2}+(y-\eta)^{2}\right]^{3 / 2}} d \xi d \eta \\
& -\frac{(1-\nu) \sigma_{0}^{2} a}{2 \pi G} \nabla \cdot\left(\frac{\nabla h}{|\nabla h|}\right) \log \left(\frac{2 \pi r_{c}|\nabla h|}{a}\right) \\
& \left.\left.-\frac{(1-\nu) \sigma_{0}^{2} a}{2 \pi G} \frac{\nabla h D^{2} h \nabla^{T} h}{|\nabla h|^{3}}\right]\right\} .
\end{aligned}
$$

The first two terms in this equation containing parameters $g_{1}$ and $g_{3}$ are due to the step line energy and the force dipole interaction between steps, respectively. The last three terms come from the long-range force monopole interaction due to the misfit stress. The anisotropic mobility tensor $D$ on a stepped surface has been obtained by Margetis and Kohn. ${ }^{42}$ In this paper, we focus on the long-range elastic effect and assume that the mobility $D$ is isotropic which corresponds to the case of no barriers for the adatom attachment to the steps. $^{42}$

Note that our continuum model is derived from a discrete model based on the simplifications of elastic isotropy and same elastic constants in the film and the substrate. The elastic effects of a step are described by the widely used approximation of force dipole on unstrained film and force monopole in the presence of the misfit. Removal of these simplifications will be considered in future work.

In this paper, we will use our continuum model to study the linear instability and evolution in the nonlinear regime for a planar surface representing a uniform straight step array. The domain size $L=N l_{t}$, where $N$ is the number of steps in the domain and $l_{t}$ is the average distance between adjacent steps. Scaling $x$ and $y$ by $\frac{N l_{t}}{2 \pi}, h$ by $\frac{N a}{2 \pi}$, and $t$ by $\frac{\left(N l_{t}\right)^{3} N a}{D g_{1}(2 \pi)^{4}}$, we have the following dimensionless evolution equation in this case: 


$$
\begin{aligned}
\frac{\partial h}{\partial t}= & \Delta\left[-\nabla \cdot\left(\frac{\nabla h}{|\nabla h|}+\alpha_{1}|\nabla h| \nabla h\right)\right. \\
& -\alpha_{2} \int_{-\infty}^{\infty} \int_{-\infty}^{\infty} \frac{(x-\xi) h_{x}(\xi, \eta)+(y-\eta) h_{y}(\xi, \eta)}{\left[(x-\xi)^{2}+(y-\eta)^{2}\right]^{3 / 2}} d \xi d \eta \\
& \left.-\alpha_{3} \nabla \cdot\left(\frac{\nabla h}{|\nabla h|}\right) \log \left(\alpha_{4}|\nabla h|\right)-\alpha_{3} \frac{\nabla h D^{2} h \nabla^{T} h}{|\nabla h|^{3}}\right],
\end{aligned}
$$

where the dimensionless constants

$$
\begin{gathered}
\alpha_{1}=\frac{g_{3} a^{2}}{g_{1} l_{t}^{2}}, \quad \alpha_{2}=\frac{(1-\nu) \sigma_{0}^{2} N a}{4 \pi^{2} g_{1} G}, \quad \alpha_{3}=\frac{(1-\nu) \sigma_{0}^{2} a}{2 \pi g_{1} G}, \\
\alpha_{4}=\frac{2 \pi r_{c}}{l_{t}} .
\end{gathered}
$$

The parameter $\alpha_{1}$ is related to the force dipole interaction and parameters $\alpha_{2}$ and $\alpha_{3}$ are from the force monopole interaction $\left(2 \pi \alpha_{2} / \alpha_{3}=N\right.$ is the number of steps in the domain). These parameters indicate the relative strength of the elastic effects with respect to the step line energy. The parameter $\alpha_{4}$ is a factor inside the logarithm term.

\section{COMPARISON WITH DISCRETE MODEL}

In this section, we examine the accuracy of our model by an example of comparison with the discrete model. Specifically, for a given stepped surface and its corresponding smooth profile, we compare the relative errors of our continuum model $\mu_{m}(x, y)$ given in Eq. (21) and the traditional continuum approximation $\mu_{m}^{0}(x, y)$ given in Eq. (15), respectively, with respect to the discrete model $\tilde{\mu}_{m}(x, y)$ given in Eqs. (18)-(20).

We consider the following smooth surface profile:

$$
h(x, y)=\frac{a}{l_{t}}\left[L-x+\epsilon L \cos \left(\frac{8 \pi x}{L}+\frac{2 \pi y}{L}\right)\right],
$$

with $\epsilon=0.01592$, and the average distance between steps $l_{t}$ $=20 \mathrm{a}$. The surface profile is a periodic function with period $L$ plus a linear function. Assume that there are $N$ steps in the domain $[0, L]^{2}$, and the location of the step $\gamma_{j}$ is the contour line of $h(x, y)$ for $h=h_{j}=j a-h_{0}, j=1,2, \ldots, N$, where $h_{0}$ is a constant. We evaluate the chemical potential at the point $(x, y)=(L / 2, L / 2)$. The constant $h_{0}$ is chosen so that this point is on a step. A surface profile and location of the steps are shown in Fig. 3.

The traditional continuum approximation $\mu_{m}^{0}(x, y)$ given by Eq. (15) can be computed using the fast-Fouriertransform (FFT) method,

$$
{\widehat{\left(\mu_{m}^{0}\right)_{k_{1}, k_{2}}}}=-\frac{(1-\nu) \sigma_{0}^{2}}{2 \pi G} L\left(k_{1}^{2}+k_{2}^{2}\right)^{1 / 2} \hat{h}_{k_{1}, k_{2}},
$$

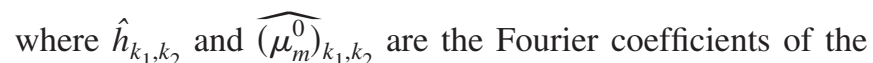
term $e^{i\left(k_{1} x+k_{2} y\right)}$ for $h(x, y)$ (the periodic part) and $\mu_{m}^{0}(x, y)$, respectively. Our continuum model $\mu_{m}(x, y)$ given in Eq. (21) can also be computed using FFT. The parameter $r_{c}$
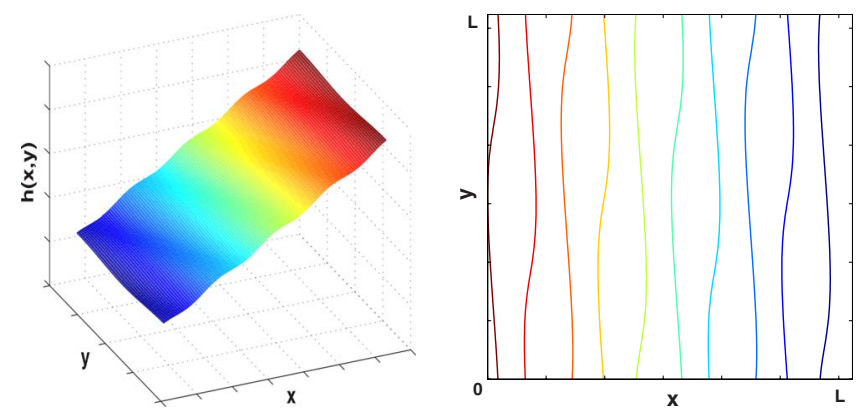

(a)

(b)

FIG. 3. (Color online) (a) The smooth surface given by Eq. (30) and (b) the location of steps for $N=10$.

$=0.2 w_{0}$, which is obtained from Eq. (22) with

$$
\delta(\omega)= \begin{cases}\frac{1}{2 w_{0}}\left(1+\cos \frac{\pi \omega}{w_{0}}\right), & -w_{0} \leq \omega \leq w_{0} \\ 0, & \text { otherwise. }\end{cases}
$$

We choose the width of the regularized delta function $w_{0}$ $=1.5 \mathrm{a}$.

Recall that the discrete model $\tilde{\mu}_{m}(x, y)$ given in Eqs. (18)-(20) can be obtained by inserting the exact stepped surface profile $\tilde{h}(x, y)$ given by Eq. (17) into Eq. (15). We use Eqs. (15) and (17) directly to compute $\tilde{\mu}_{m}(x, y)$, using the unit normal vector $\mathbf{n}=-\nabla h(x, y) /|\nabla h(x, y)|$, and the regularized delta function in Eq. (32) with the variable $\omega$ replaced by the distance from a point on the surface to the nearby step $\gamma_{j}$,

$$
\begin{aligned}
d= & -\frac{h-h_{j}}{\left(h_{x}^{2}+h_{y}^{2}\right)^{1 / 2}}-\frac{1}{2} \frac{h_{x}^{2} h_{x x}+2 h_{x} h_{y} h_{x y}+h_{y}^{2} h_{y y}}{\left(h_{x}^{2}+h_{y}^{2}\right)^{5 / 2}}\left(h-h_{j}\right)^{2} \\
& +O\left(\left(h-h_{j}\right)^{3}\right) .
\end{aligned}
$$

The derivation of this formula can be found in Appendix A in Ref. 47. Then we can also use FFT to compute the discrete model $\tilde{\mu}_{m}(x, y)$.

Figure 4 shows the relative errors of our continuum model $\mu_{m}(x, y)$ and the traditional continuum approximation $\mu_{m}^{0}(x, y)$, respectively, with respect to the discrete model $\tilde{\mu}_{m}(x, y)$, at the point $(x, y)=(L / 2, L / 2)$ with different values of $N$. From the figure, we can see that as a continuum approximation to the discrete model $\tilde{\mu}_{m}(x, y)$, the error of our model $\mu_{m}(x, y)$ is much smaller than that of the traditional approximation $\mu_{m}^{0}(x, y)$. When $N=10$, our model gives relative error $0.7 \%$, while the relative error of the traditional continuum model is 52\%. This example shows that our continuum model $\mu_{m}(x, y)$ given in Eq. (21) is more accurate than the traditional continuum approximation $\mu_{m}^{0}(x, y)$ given in Eq. (15).

Note that in this example of comparison, we compute the discrete model $\tilde{\mu}_{m}(x, y)$ using Eqs. (15) and (17) and a regularized delta function in Eq. (32). In this formulation, the line integrals in $\tilde{\mu}_{m}^{\text {int }}(x, y)$ in Eq. (19) are approximated by convolutions with the regularized delta function, as in $\tilde{\mu}_{m}^{\text {self }}(x, y)$ in Eq. (20). Convergence tests as the width of the regularized delta function goes to 0 show that the relative error of this 


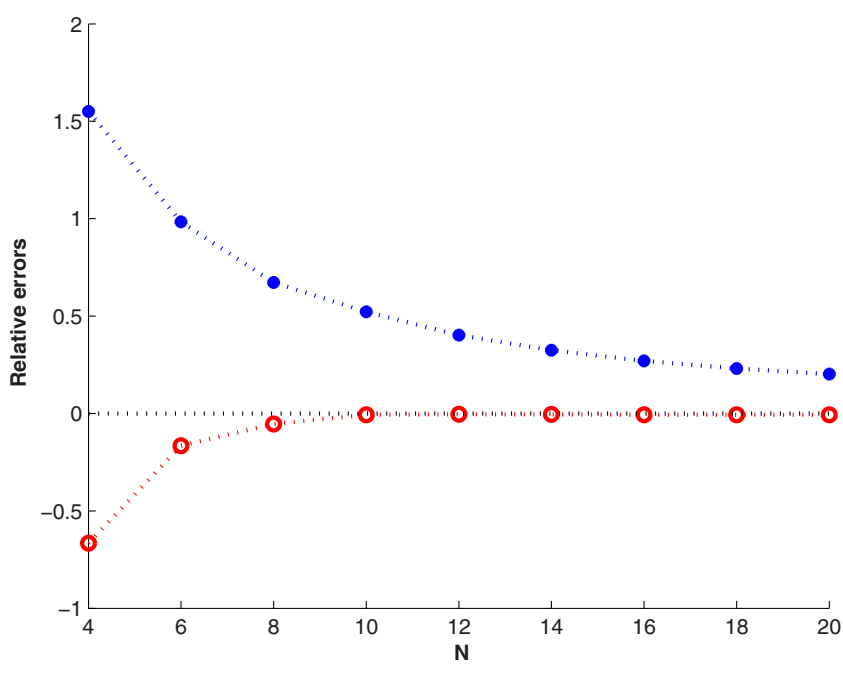

FIG. 4. (Color online) The relative errors of our continuum model $\mu_{m}(x, y)$ and the traditional continuum approximation $\mu_{m}^{0}(x, y)$, respectively, with respect to the discrete model $\tilde{\mu}_{m}(x, y)$, at the point $(x, y)=(L / 2, L / 2)$ with different values of $N$. The (red) line with circles shows the relative errors using our continuum model $\mu_{m}(x, y)$. The (blue) line with dots shows the relative errors using the traditional continuum approximation $\mu_{m}^{0}(x, y)$.

approximation is less than $2 \%$, which is much smaller than the relative error of the traditional continuum approximation $\mu_{m}^{0}(x, y)$ (see Fig. 4). Thus this numerical approximation does not change the conclusion of the comparison.

\section{LINEAR INSTABILITY}

In this section, we use our continuum equation in Eq. (28) to study the linear instability of a planar surface (representing a uniform straight step array) to small perturbations. Consider

$$
h(x, y, t)=-x+\varepsilon e^{i k_{1} x+i k_{2} y+\omega t}
$$

in the length units of Eq. (28), where the amplitude of the perturbation $\varepsilon$ is very small. Inserting this expression into Eq. (28) and keeping the $O(\varepsilon)$ terms, we obtain the following dispersion relation:

$$
\begin{aligned}
\omega= & \left(k_{1}^{2}+k_{2}^{2}\right)\left[-\left(2 \alpha_{1}+\alpha_{3}\right) k_{1}^{2}-\left(1+\alpha_{1}+\alpha_{3} \log \alpha_{4}\right) k_{2}^{2}\right. \\
& \left.+2 \pi \alpha_{2}\left(k_{1}^{2}+k_{2}^{2}\right)^{1 / 2}\right],
\end{aligned}
$$

where the parameters are given in Eq. (29). When $\omega<0$, the
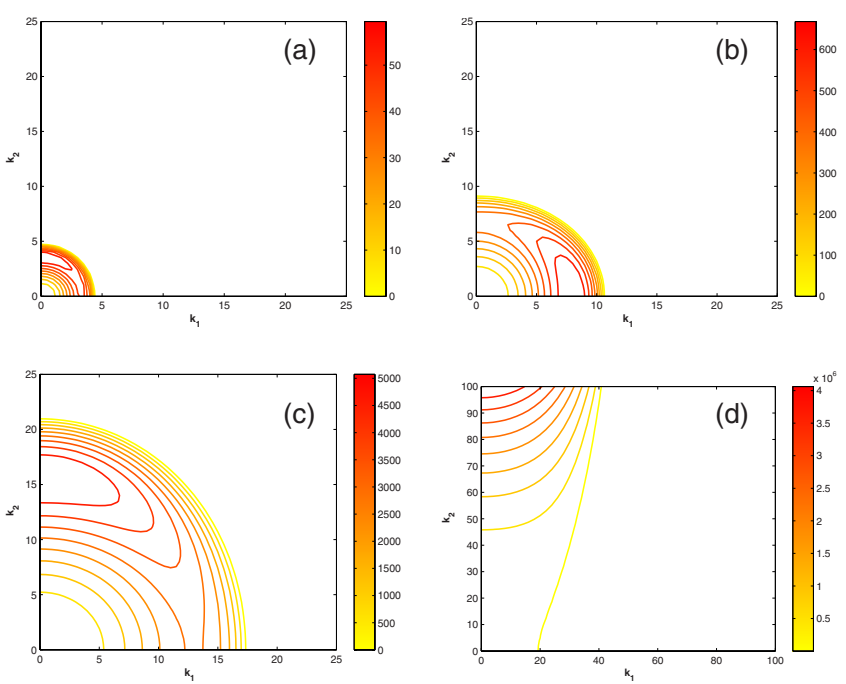

FIG. 5. (Color online) Dispersion relation $\omega\left(k_{1}, k_{2}\right)$ for $g_{3} / g_{1}$ $=286$ and different values of $l_{t}$ : (a) $l_{t}=25 a$; (b) $l_{t}=50 a$; (c) $l_{t}$ $=120 a$; (d) $l_{t}=280 a$. The contour lines are plotted in the unstable region where $\omega>0$. Since $\omega\left(k_{1}, k_{2}\right)$ is an even function, only the region $k_{1}, k_{2}>0$ is shown.

planar surface is stable to the small perturbation with mode $\left(k_{1}, k_{2}\right)$; when $\omega>0$, the planar surface is unstable to the small perturbation with this mode.

The last term in the dispersion relation in Eq. (35) is a destabilizing term, which is from the integral term in the evolution equation (28) and is the main effect of the force monopole interaction due to the misfit. This destabilizing effect is the same as that in the classical stress-driven morphology instability ${ }^{6-10}$ The step line energy, which gives the term -1 in the coefficient of $k_{2}^{2}$ inside the brackets in the dispersion relation, and the force dipole interaction, which gives the terms containing $\alpha_{1}$ in the dispersion relation, are both stabilizing. The additional nonlinear terms due to the misfit force monopole interaction give the two terms containing $\alpha_{3}$ in the dispersion relation, which modify the instability.

Figure 5 shows some examples of this dispersion relation. The parameters are chosen as follows: $g_{1}=0.03 \mathrm{~J} / \mathrm{m}^{2}, g_{3}$ $=8.58 \mathrm{~J} / \mathrm{m}^{2}\left(g_{3} / g_{1}=286\right),{ }^{52}$ the step height $a=0.27 \mathrm{~nm}$, the elastic moduli $\nu=0.25$ and $G=3.8 \times 10^{10} \mathrm{~Pa}$, and the misfit $\varepsilon_{0}=0.024$. The core parameter $r_{c}$ of a step is assumed to be $a$. The number of steps in a period $N=20$. Figure 5(a) shows the dispersion relation when the distance between adjacent steps $l_{t}=25 a$. We can see that the unstable region, i.e., the
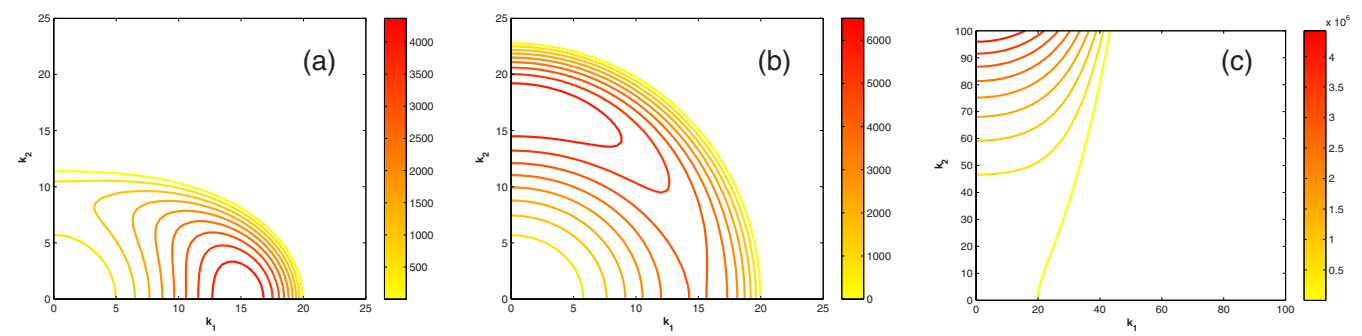

FIG. 6. (Color online) Dispersion relation $\omega\left(k_{1}, k_{2}\right)$ for $g_{3} / g_{1}=1$ and different values of $l_{t}$ : (a) $l_{t}=50 a$; (b) $l_{t}=120 a$; (c) $l_{t}=280 a$. The contour lines are plotted in the unstable region where $\omega>0$. Since $\omega\left(k_{1}, k_{2}\right)$ is an even function, only the region $k_{1}, k_{2}>0$ is shown. 
region where $\omega$ is positive, is a bounded region near the origin, which means small wave-number instability. We can also see from Fig. 5(a) that the most unstable mode of $\omega$ is attained on the $k_{2}$ axis, which implies that in this case the dominant instability is step undulation. Figure 5(b) shows the dispersion relation when $l_{t}=50 a$, in which the instability also occurs at small wave numbers. The most unstable mode of $\omega$ is attained on the $k_{1}$ axis, which implies that in this case the dominant instability is step bunching. The dispersion relation for the case $l_{t}=120 a$ shown in Fig. 5(c) is qualitatively similar to that for the case $l_{t}=25 a$ shown in Fig. 5(a). When $l_{t}$ is very large, as shown in Fig. 5(d) for the case $l_{t}=280 a$, the unstable region is unbounded in $k_{2}$ direction, and the positive value of $\omega$ is unbounded as $k_{2}$ increases along the $k_{2}$ axis. This means that the undulation instability occurs for any small perturbation along the steps.

Examples of the dispersion relation for small ratio of $g_{3} / g_{1}$ are shown in Fig. 6, where $g_{1}=0.03 \mathrm{~J} / \mathrm{m}^{2}, g_{3}$ $=0.03 \mathrm{~J} / \mathrm{m}^{2}$, i.e., $g_{3} / g_{1}=1$, and other parameters are the same as those in Fig. 5.

In the above examples, we see transitions between step bunching instability and step undulation instability and transitions between bounded unstable region to unbounded unstable region. The step bunching instability dominates when the coefficient $2 \alpha_{1}+\alpha_{3}$ of the $k_{1}^{2}$ term is less than the coefficient $1+\alpha_{1}+\alpha_{3} \log \alpha_{4}$ of the $k_{2}^{2}$ term in the dispersion relation in Eq. (35); when the former is greater, step undulation instability dominates. The unstable region changes from bounded to unbounded when the coefficient $1+\alpha_{1}$ $+\alpha_{3} \log \alpha_{4}$ of the $k_{2}^{2}$ term in the dispersion relation changes its sign from positive to negative.

These transitions are summarized in Fig. 7 in terms of the distance between adjacent steps $l_{t}^{53}$ and the relative value of the misfit $\varepsilon_{0}$ with respect to $\left[(1-\nu) \pi g_{1} / 2(1+\nu)^{2} G a\right]^{1 / 2}$, for fixed value of the ratio $g_{3} / g_{1}$. Figure 7(a) shows the case of a large ratio $g_{3} / g_{1}: g_{3} / g_{1}=286$. For a relative small misfit, the horizontal constant-misfit line intersects the boundary between step bunching dominant region and step undulation dominant region twice. As $l_{t}$ increases, the dominant instability changes from step undulation to step bunching and then back to step undulation. For a very large $l_{t}$, any perturbation in the direction along the steps causes instability. See Fig. 5 for the dispersion relation for different values of $l_{t}$ in this case (where $\left.\varepsilon_{0} /\left[(1-\nu) \pi g_{1} / 2(1+\nu)^{2} G a\right]^{1 / 2}=0.51\right)$. For a relative large misfit in Fig. 7(a), the step undulation instability always dominates. For a small $l_{t}$, only perturbations with small wave numbers in the direction along the steps cause the step undulation instability; while for a large $l_{t}$, any perturbation in the direction along the steps is unstable. Figure 7(b) shows the case of a small ratio $g_{3} / g_{1}: g_{3} / g_{1}=1$. In Fig. 7(b), any horizontal line of constant misfit intersects the boundary between step bunching dominant region and step undulation dominant region once. As $l_{t}$ increases, the dominant instability changes from step bunching to step undulation, and for a very large $l_{t}$, any perturbation in the direction along the steps causes instability. See Fig. 6 for the dispersion relation for different values of $l_{t}$ in this case. The transition from step bunching instability to step undulation instability as $l_{t}$ increases has been observed in experiments ${ }^{36}$ and is shown using discrete step dynamics models. ${ }^{23,29}$
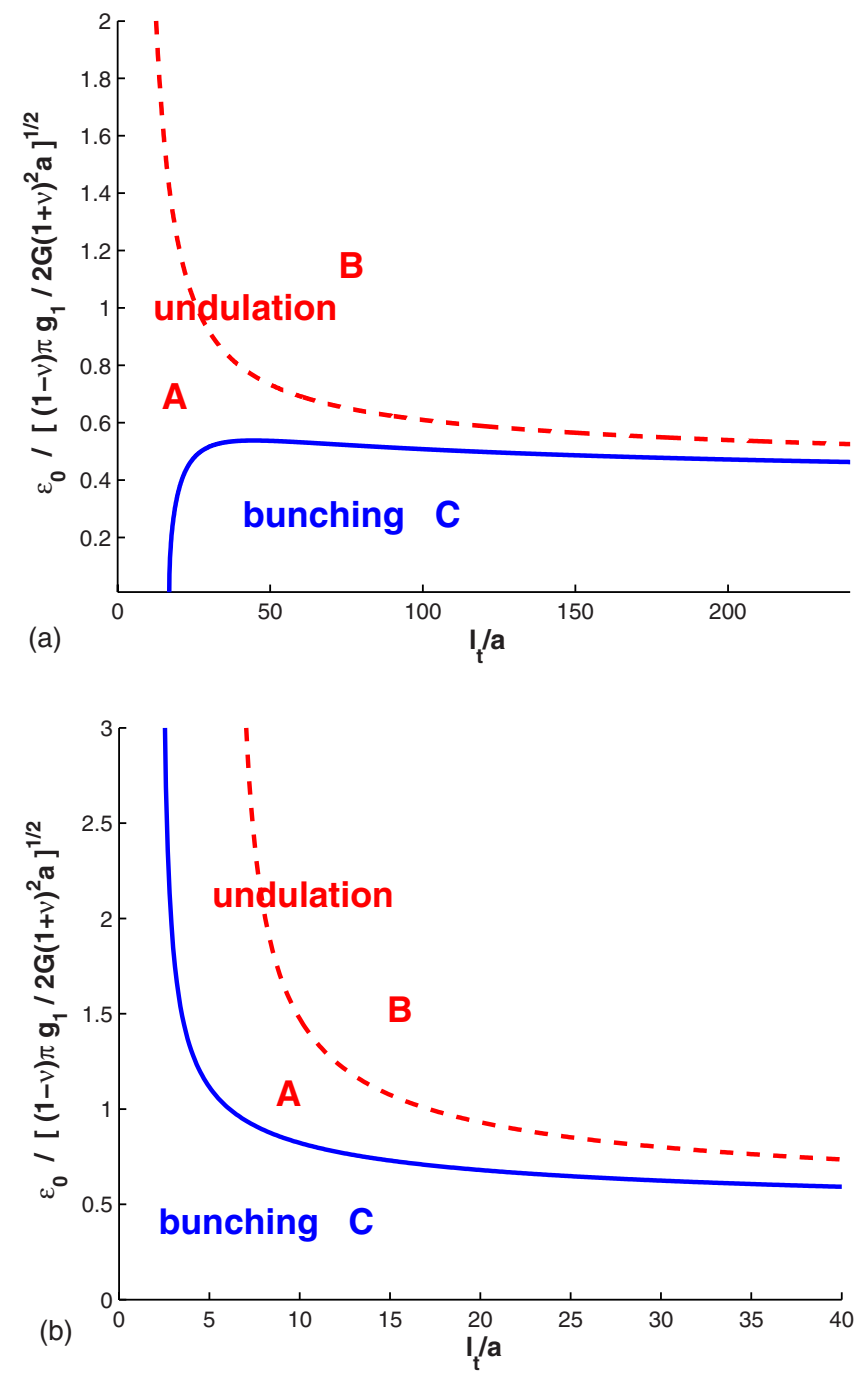

FIG. 7. (Color online) Competing step instabilities with respect to the distance between adjacent steps $l_{t}$ and the relative value of the misfit for two different values of the ratio $g_{3} / g_{1}$ : (a) $g_{3} / g_{1}=286$ and (b) $g_{3} / g_{1}=1$. In region $\mathrm{C}$, the step bunching instability dominates, and in regions $\mathrm{A}$ and $\mathrm{B}$, the step undulation instability dominates. In region $\mathrm{A}$ in the step undulation regime, only perturbations with small wave numbers in the direction along the steps cause the step undulation instability [e.g., the dispersion relation in Figs. 5(a) and $5(\mathrm{c})]$. In region B in the step undulation regime, any perturbation in the direction along the steps is unstable [e.g., the dispersion relation in Fig. 5(d)]. Both the boundary between the bunching and undulation regions and that between regions $\mathrm{A}$ and $\mathrm{B}$ approach zero-misfit axis as $l_{t} \rightarrow \infty$. The boundary between regions $\mathrm{A}$ and $\mathrm{B}$ approaches $l_{t} / a=2 \pi r_{c} / a$ as the relative misfit goes to infinity. In case (a), the boundary between bunching and undulation intersects the zeromisfit axis at $l_{t} / a=\sqrt{g_{3} / g_{1}}$, while in case (b), this boundary approaches $l_{t} / a=2 \pi r_{c} /(e a)$ as the relative misfit goes to infinity. Note that there is no instability on the zero-misfit axis.

Finally, we compare our linear instability results with those using the traditional continuum approximation $\mu_{m}^{0}(x, y)$ in Eq. (15), which corresponds to the case where the terms containing $\alpha_{3}$ are removed in our dispersion relation in Eq. (35). Figure 8 shows the dispersion relation using $\mu_{m}^{0}(x, y)$ when all the parameters are the same as those in the disper- 


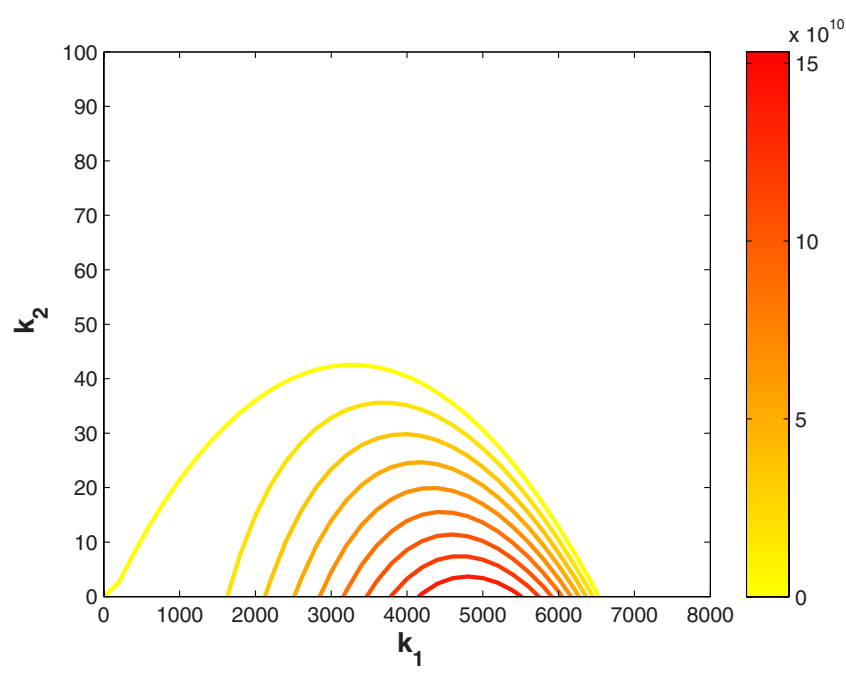

FIG. 8. (Color online) Dispersion relation using the traditional continuum approximation $\mu_{m}^{0}(x, y)$ in Eq. (15) when $g_{3} / g_{1}=1$. The dispersion relation is qualitatively similar for all $l_{t}$. This plot shows the one with $l_{t}=50 a$.

sion relation using our model shown in Fig. 6. Comparing Fig. 8 with Fig. 6, we can see that by using the traditional continuum approximation $\mu_{m}^{0}(x, y)$, the step bunching instability always dominates and there is no transition from step bunching instability to step undulation instability as $l_{t}$ increases, which does not agree with the experimental observations $^{36}$ and the analytical results using discrete step dynamics models. ${ }^{23,29}$ This comparison shows that the additional nonlinear terms in our continuum model are crucial to include the discrete features of the stepped surface.

\section{EXAMPLES OF NONLINEAR EVOLUTION}

In this section, we present some examples of evolution of the stepped surfaces in the nonlinear regime using our continuum model (28) with dimensionless constants in Eq. (29). We focus on the surface morphology in the early stage of nonlinear regime.

In the simulations, we choose $g_{1}=0.03 \mathrm{~J} / \mathrm{m}^{2}, g_{3}$ $=8.58 \mathrm{~J} / \mathrm{m}^{2}$, the step height $a=0.27 \mathrm{~nm}$, the elastic moduli $\nu=0.25$, and $G=3.8 \times 10^{10} \mathrm{~Pa}$. The core parameter $r_{c}$ of a step is assumed to be $a$.

We use the pseudospectral method with the FFT (Ref. 54) under periodic boundary conditions in the simulations. For the time discretization, we use the trapezoid rule for the linear equation obtained in the linear instability analysis, and the forward Euler method for the remaining part after the linear equation is subtracted from the full nonlinear equation. The simulation domain is $[0,2 \pi] \times[0,2 \pi]$ divided into $128 \times 128$ grid points. Initially, the surface is planar subject to some small perturbations with wave numbers near $\left(k_{1}, k_{2}\right)$,

$$
\begin{aligned}
h(x, y)= & -x+\sum_{\left|k_{1}^{\prime}-k_{1}\right| \leq k_{1}^{0},\left|k_{2}^{\prime}-k_{2}\right| \leq k_{2}^{0}} \frac{10^{-3}}{\left[\left(k_{1}^{\prime}-k_{1}\right)^{2}+\left(k_{2}^{\prime}-k_{2}\right)^{2}\right]^{3 / 2}+1} \\
& \times \cos \left(k_{1}^{\prime} x+k_{2}^{\prime} y\right),
\end{aligned}
$$

where $\left(k_{1}, k_{2}\right)$ is a wave-number pair in the unstable region in linear instability analysis, and $k_{1}^{0}, k_{2}^{0}=1$ or 2 .
Figure 9 shows the evolution of a surface with parameters $\varepsilon_{0}=0.012, N=40, l_{t}=50 a$, and $\left(k_{1}, k_{2}\right)=(7,1)$ in the initial perturbations in Eq. (36). We plot the surface profiles, the contour lines of the surface height which indicate the locations of steps, and a cross section of the surface for some constant value of $y$. According to the linear instability analysis, the step bunching instability dominates in this case. In the very early stages of the evolution, both perturbations of step bunching and step undulation grow [see Figs. 9(d)-9(f)], which agrees with the linear instability analysis. Then step bunching continues, and the steps become more and more straight due to the nonlinear effect [see Figs. 9(g)-9(1)]. After all the steps become straight and only the step bunching instability remains [see Figs. 9(m)-9(o)], we can see a rearrangement of step bunches [e.g., a contour line between $x$ $=2$ and $x=3$ moves from the bunch near $x=3$ to the bunch near $x=2$ in Figs. 9(m)-9(o)].

Figure 10 shows the evolution of a surface with parameters $\varepsilon_{0}=0.024, N=10, l_{t}=50 a$, and $\left(k_{1}, k_{2}\right)=(4,2)$ in the initial perturbations in Eq. (36). As in the previous example, the step bunching instability is also dominant according to the linear instability analysis, and both perturbations of step bunching and step undulation grow in the very early stages of the simulation. However, unlike in the previous example, the steps do not become straight in this case. This can be understood by the fact that in this case, the misfit $\varepsilon_{0}$ is larger than that in the previous example, and the destabilizing effect of the misfit energy is stronger. It can also be seen that in the last state shown in Fig. 10, the surface is divided into regions where the steps are almost straight and step density is high and regions where step density is low. If we focus on a cross section of the surface along constant $y$, as shown in the right column of the images, the surface still has the step bunching morphology.

Figure 11 shows the evolution of a surface with parameters $\varepsilon_{0}=0.024, N=20, l_{t}=25 a$, and $\left(k_{1}, k_{2}\right)=(3,3)$ in the initial perturbations in Eq. (36). In this case, step undulation instability is dominant according to the linear instability analysis. We can see from the simulation that perturbations of step undulation grow and no obvious step bunching instability is observed. However, if we focus on a cross section of the surface along constant $y$, as shown in the right column of the images in Fig. 11, the surface still has the step bunching morphology.

We note that the final states in these simulations are not equilibrium states. We stop the simulations when the equation becomes highly singular which makes simulations very difficult. Developing efficient numerical schemes will be done in future work.

In summary of this section, we see surface morphologies of step bunching, step undulation, and combination of them in the early stage of evolution of the surface in the nonlinear regime in our simulations. It is interesting to see that along a cross section perpendicular to the initially almost straight steps, the surface takes the step bunching morphology even though the overall two-dimensional morphology is step undulation or combination of step bunching and undulation.

If we remove the two nonlinear terms with coefficient $\alpha_{3}$ in the evolution equation in Eq. (28), it is very difficult to obtain the step bunching morphology as shown in Fig. 9. 


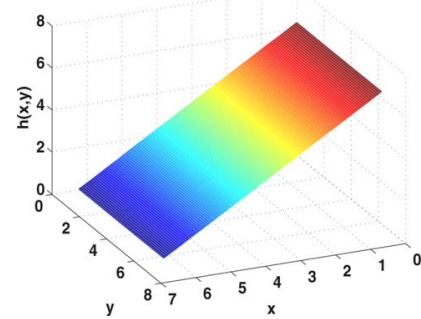

(a)

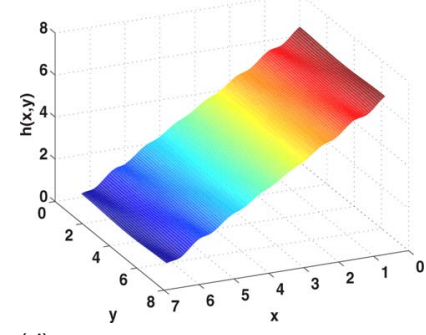

(d)

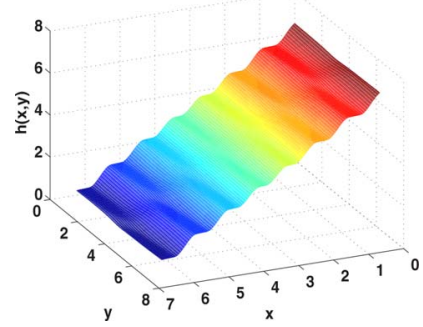

(g)

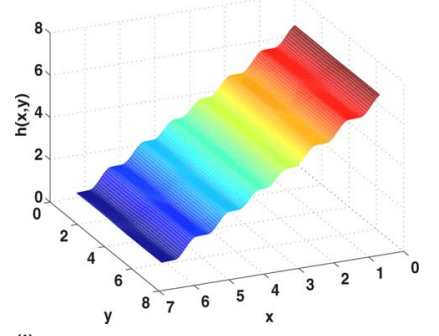

(j)

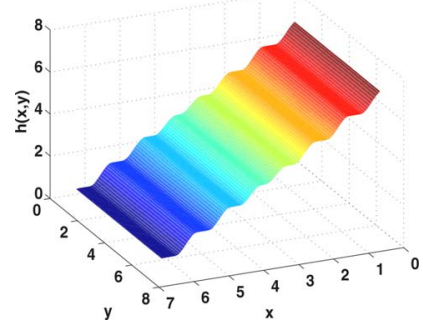

(m)

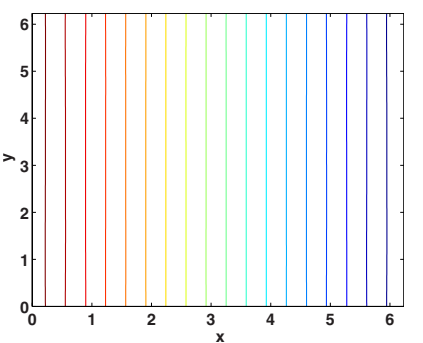

(b)

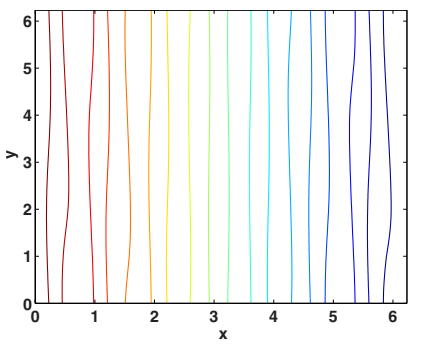

(e)

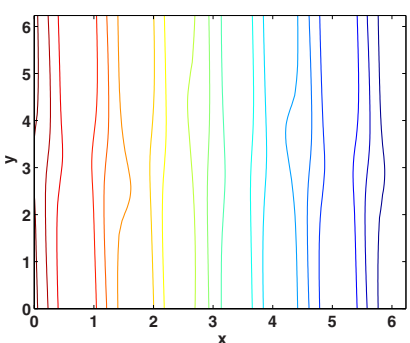

(h)

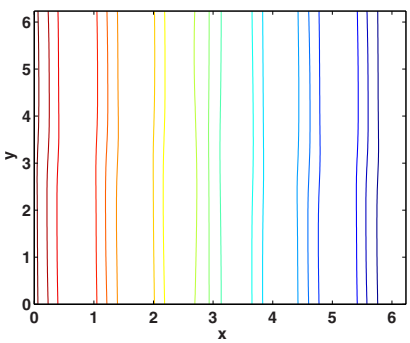

(k)

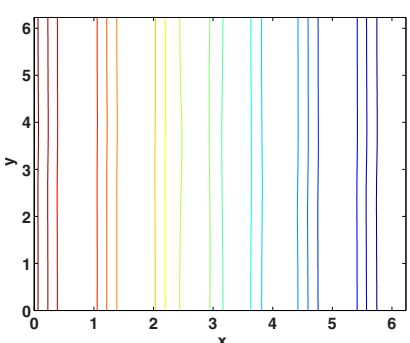

(n)

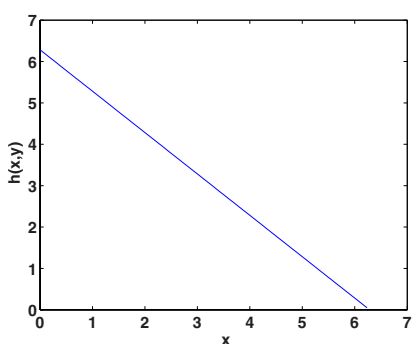

(c)

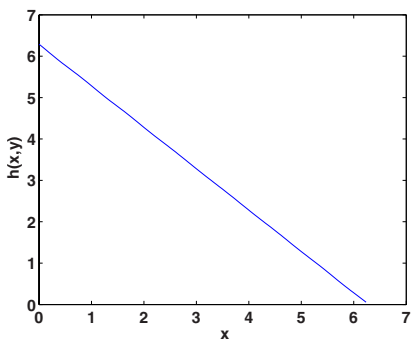

(f)

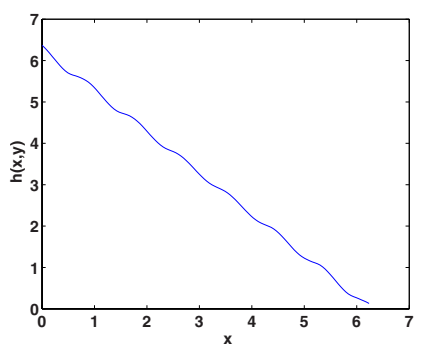

(i)

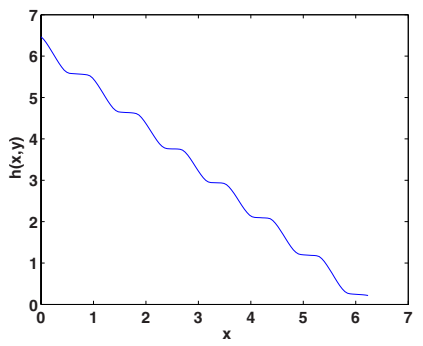

(I)

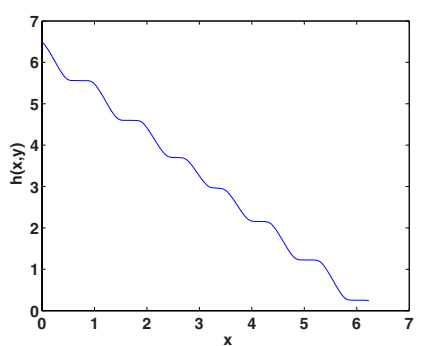

(o)

FIG. 9. (Color online) Time evolution of the surface with parameters $\varepsilon_{0}=0.012, N=40, l_{t}=50 a$, and $\left(k_{1}, k_{2}\right)=(7,1)$. (a) - (c): $t=0$; (d) $-(\mathrm{f})$ $t=0.0225$; (g)-(i): $t=0.0315$; (j)-(1): $t=0.0395$; (m) $-(\mathrm{o}) t=0.0504$. The left column of images shows the three-dimensional view, the column of images in the middle shows the contour lines of the surface height which indicate the locations of steps, and the right column of images shows the cross section of the surface at $y=\pi$.

This can be understood by the dispersion relation in Eq. (35) as follows. When the surface is flat, locally $l_{t}$ is large and $\alpha_{1}$ is small [see Eq. (29)]. If the terms with coefficient $\alpha_{3}$ are removed, the stabilizing effect in the direction normal to the almost straight step becomes very weak thus it is difficult to maintain the step bunching surface morphology. This shows that the additional nonlinear terms we obtained besides the traditional continuum expression are also crucial to 


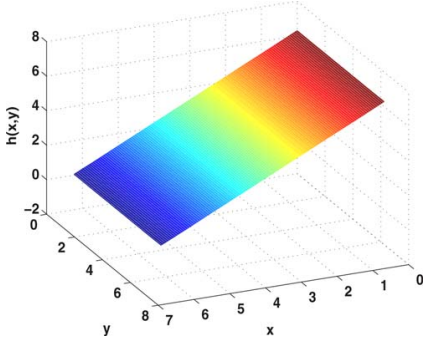

(a)

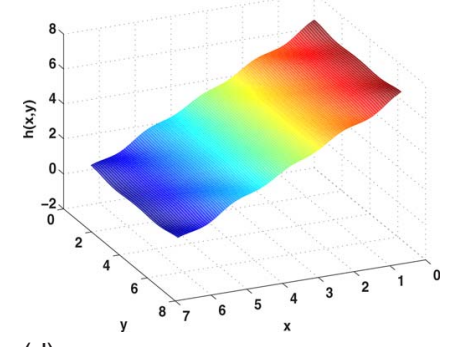

(d)

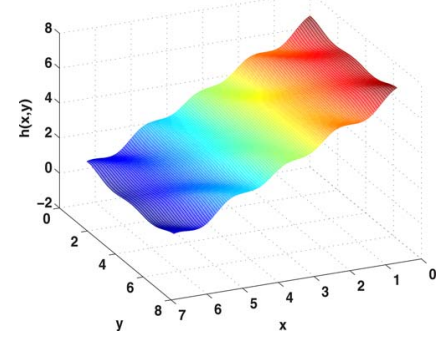

(g)

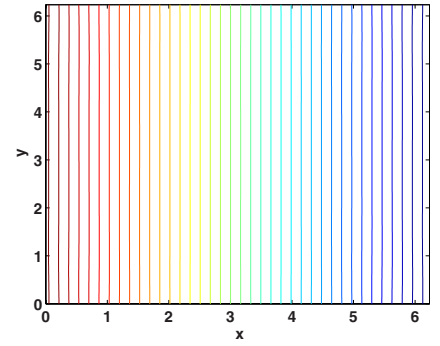

(b)

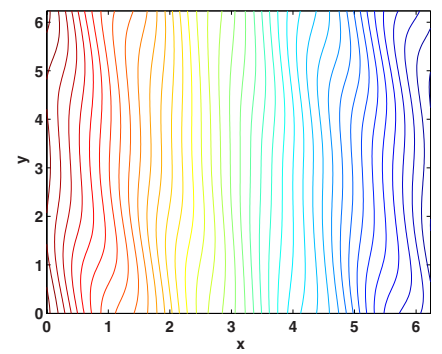

(e)

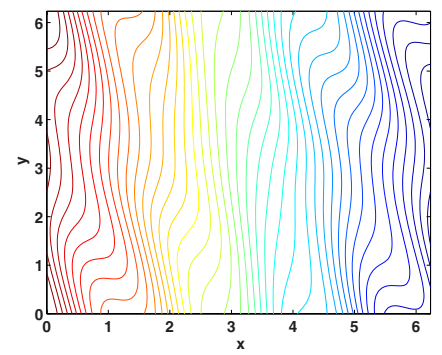

(h)

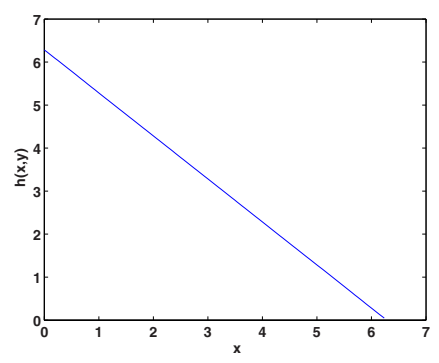

(c)

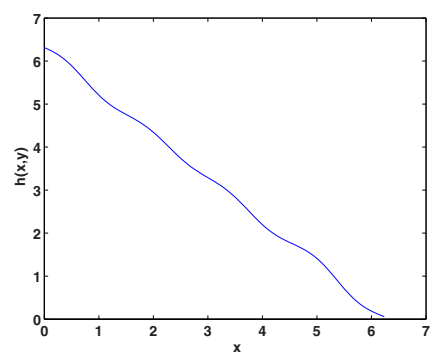

(f)

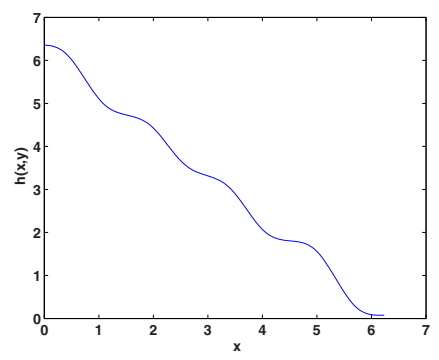

(i)

FIG. 10. (Color online) Time evolution of the surface with parameters $\varepsilon_{0}=0.024, N=10, l_{t}=50 a$, and $\left(k_{1}, k_{2}\right)=(4,2)$. (a) $-(\mathrm{c})$ : $t=0$; (d) $-(\mathrm{f})$ : $t=0.12$; (g)-(i): $t=0.1468$. The left column of images shows the three-dimensional view, the column of images in the middle shows the contour lines of the surface height which indicate the locations of steps, and the right column of images shows the cross section of the surface at $y=5.4$.

describe the morphology of stepped surfaces under elastic effects in the nonlinear regime of evolution in $2+1$ dimensions, as the nonlinear term in the 1+1-dimensional model in Ref. 35.

\section{CONCLUSIONS}

In this paper, we present a 2+1-dimensional continuum model for the long-range elastic interaction on stepped surface of a strained film. The continuum model is derived rigorously from the discrete model for the interaction between steps; thus it incorporates the discrete features of the stepped surfaces. Examples of comparison show that our continuum model, as an approximation to the discrete model, is much more accurate than the traditional continuum approximation. In the linear instability of a planar surface, our continuum model gives the transition from step bunching instability to step undulation instability as the distance between adjacent steps increases, which agrees with the experimental observations and the results of discrete models, and which is missing using the traditional continuum approximation. Numerical simulations of the surface evolution in the nonlinear regime using our model successfully show surface morphologies of step bunching, step undulation, and their combination. The additional nonlinear terms we obtained besides the traditional continuum expression are shown also to be crucial to describe the step bunching surface morphology in the nonlinear regime of the surface evolution.

Future work may include generalization of our continuum model under elastic anisotropy or other general conditions and investigation on surface evolution using our continuum model with anisotropic mobility ${ }^{42}$ or nucleation of steps. ${ }^{19,23,33,46,55}$

\section{ACKNOWLEDGMENTS}

This work was partially supported by Hong Kong RGC Competitive Earmarked Research under Grant No. 604604 and UGC Targets of Opportunity under Grant No. AoE-MG/ $\mathrm{P} 05 / 06$.

\section{APPENDIX: DERIVATION OF THE CONTINUUM MODEL FOR A CONICAL-STEPPED SURFACE}

In this appendix, we present a derivation of the continuum model for an axisymmetric conical-moundlike-stepped sur- 


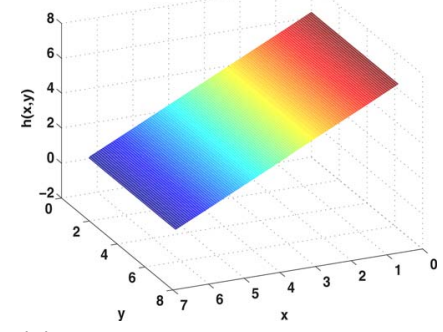

(a)

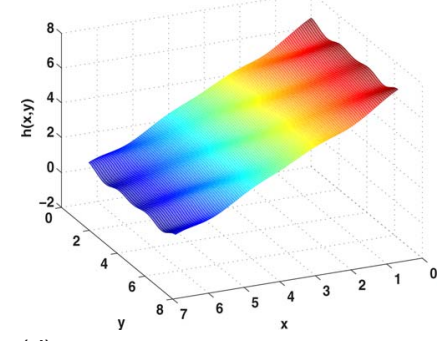

(d)

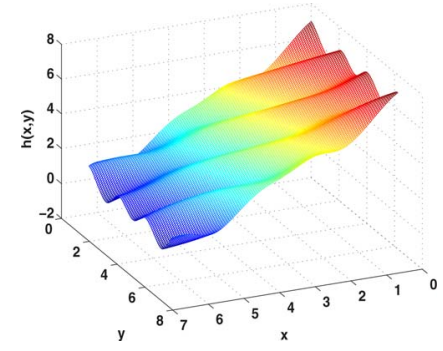

(g)

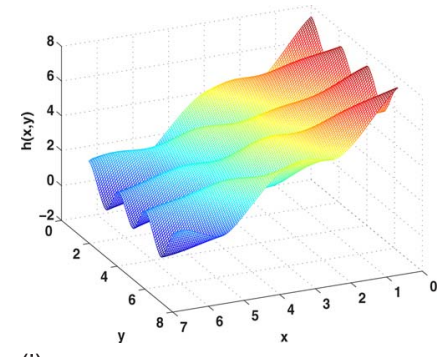

(j)

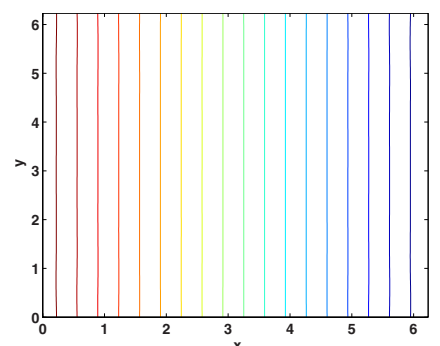

(b)

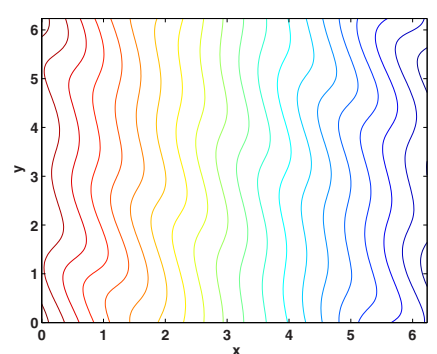

(e)

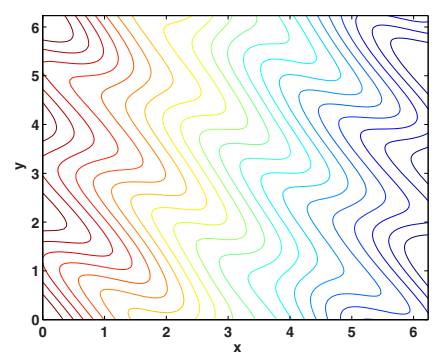

(h)

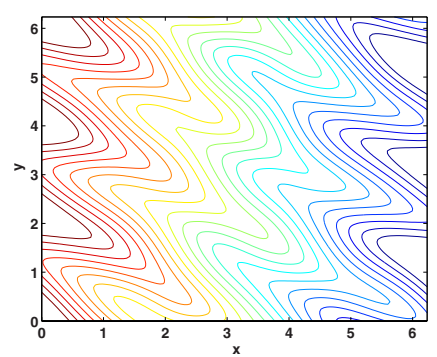

(k)

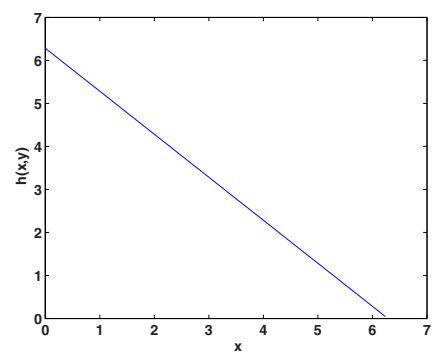

(c)

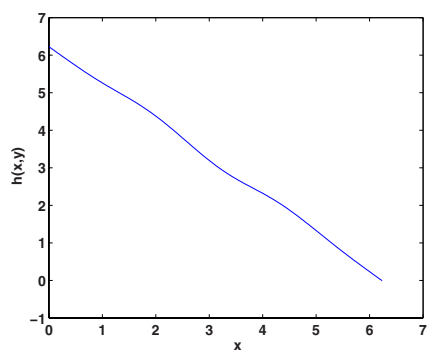

(f)

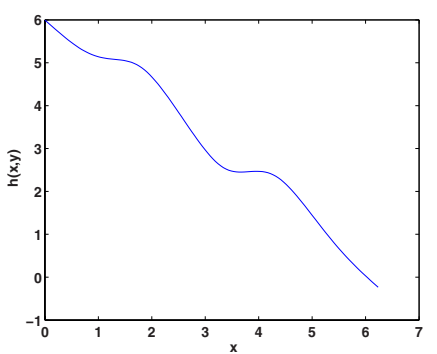

(i)

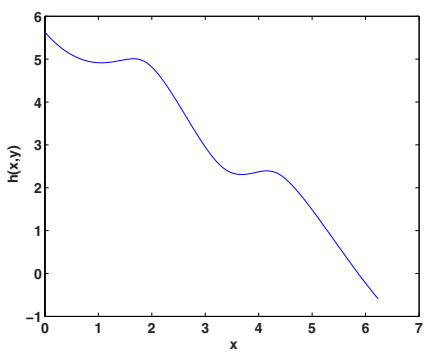

(I)

FIG. 11. (Color online) Time evolution of the surface with parameters $\varepsilon_{0}=0.024, N=20, l_{t}=25 a$, and $\left(k_{1}, k_{2}\right)=(3,3)$. (a)-(c): $t=0$; (d) $-(\mathrm{f})$ : $t=0.096$; (g)-(i): $t=0.126$; (j)-(l): $t=0.213$. The left column of images shows the three-dimensional view, the column of images in the middle shows the contour lines of the surface height which indicate the locations of steps, and the right column of images shows the cross section of the surface at $y=\pi$.

face, which consists of concentric circular steps separating flat annular terraces as illustrated in Fig. 12. The circular steps are labeled $\gamma_{j}$ with radius $r_{j}, j=1,2,3 \ldots$, respectively,

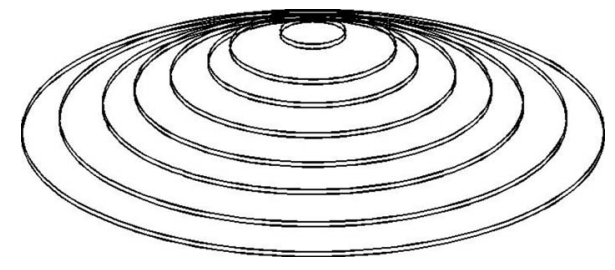

FIG. 12. A conical-stepped surface. from the top of the surface. A point $\mathbf{X}=(x, y)$ on step $\gamma_{j}$ can be written as $(x, y)=\left(r_{j} \cos \theta_{j}, r_{j} \sin \theta_{j}\right)$, where $\theta_{j}$ is the angular coordinate of $\gamma_{j}$. Then the normal direction of the step $\gamma_{j}$ at this point is $\mathbf{n}_{j}=\left(\cos \theta_{j}, \sin \theta_{j}\right)$.

In the discrete model for the long-range elastic interaction between steps, at a point on step $\gamma_{n}$ on this axisymmetric surface, the chemical potential given by Eqs. (18)-(20) can be written as

$$
\tilde{\mu}_{m}^{\text {int }}\left(r_{n}\right)=-\frac{(1-\nu) \sigma_{0}^{2} a}{\pi G} \sum_{j \neq n}\left[\frac{K\left(m_{j}\right)}{r_{j}+r_{n}}+\frac{E\left(m_{j}\right)}{r_{j}-r_{n}}\right]
$$

and 


$$
\tilde{\mu}_{m}^{\text {self }}\left(r_{n}\right)=-\frac{(1-\nu) \sigma_{0}^{2} a}{\pi G} \int_{-\infty}^{\infty} \delta(\omega)\left[\frac{K\left(m_{\omega}\right)}{\omega+2 r_{n}}+\frac{E\left(m_{\omega}\right)}{\omega}\right] d \omega,
$$

where

$$
K(m)=\int_{0}^{\pi / 2} \frac{d \theta}{\sqrt{1-m \sin ^{2} \theta}}
$$

and

$$
E(m)=\int_{0}^{\pi / 2} \sqrt{1-m \sin ^{2} \theta} d \theta
$$

are the complete elliptic integrals of the first and second kinds, respectively,

$$
m_{j}=\frac{4 r_{n} r_{j}}{\left(r_{n}+r_{j}\right)^{2}}
$$

and

$$
m_{\omega}=\frac{4 r_{n}\left(r_{n}+\omega\right)}{\left[r_{n}+\left(r_{n}+\omega\right)\right]^{2}}
$$

in Eqs. (A1) and (A2), respectively. As described in Sec. III, the delta function $\delta(\omega)$ in Eq. (A2) is a regularized Dirac delta function whose width of regularization is of the order of the lattice constant $a$. We further assume that $\delta(\omega)$ has compact support and $\delta(-\omega)=\delta(\omega)$. By Eqs. (A1) and (A2), the total chemical potential given by Eq. (18) is

$$
\begin{aligned}
\tilde{\mu}_{m}\left(r_{n}\right)= & -\frac{(1-\nu) \sigma_{0}^{2} a}{\pi G} \sum_{j \neq n}\left[\frac{K\left(m_{j}\right)}{r_{j}+r_{n}}+\frac{E\left(m_{j}\right)}{r_{j}-r_{n}}\right] \\
& -\frac{(1-\nu) \sigma_{0}^{2} a}{\pi G} \int_{-\infty}^{\infty} \delta(\omega)\left[\frac{K\left(m_{\omega}\right)}{\omega+2 r_{n}}+\frac{E\left(m_{\omega}\right)}{\omega}\right] d \omega .
\end{aligned}
$$

Although Eq. (A7) gives an exact expression for the misfit-induced long-range elastic interaction on a conicalstepped surface, in the framework of a continuum model, the conical-stepped-surface is described by a smooth profile $h(r)$ without resolving the details of the steps. The continuum approximation of the chemical potential given by Eq. (15) for this conical surface can be written as

$$
\mu_{m}^{0}\left(r_{n}\right)=\frac{(1-\nu) \sigma_{0}^{2}}{\pi G} \int_{0}^{\infty} h^{\prime}(r)\left[\frac{K(m)}{r+r_{n}}+\frac{E(m)}{r-r_{n}}\right] d r,
$$

where

$$
m=\frac{4 r_{n} r}{\left(r_{n}+r\right)^{2}} .
$$

However, this expression does not incorporate the discrete features of the stepped surface, as discussed in Secs. I and II.

In order to obtain a more accurate continuum approximation, we first rewrite the above integral $\mu_{m}^{0}\left(r_{n}\right)$ in Eq. (A8) as

$$
\mu_{m}^{0}\left(h_{n}\right)=-\frac{(1-\nu) \sigma_{0}^{2}}{\pi G} \int_{0}^{H}\left[\frac{K(m)}{r(h)+r\left(h_{n}\right)}+\frac{E(m)}{r(h)-r\left(h_{n}\right)}\right] d h,
$$

by taking $h$ instead of $r$ as the independent variable, where $H$ is the height of the conical surface which is assumed to be very large compared with the lattice constant $a$, and $r(H)$ $=0$. Then the discrete model $\widetilde{\mu}_{m}\left(r_{n}\right)$ given by Eq. (A7) can be regarded as a numerical scheme of the continuum expression $\mu_{m}^{0}\left(h_{n}\right)$ given by Eq. (A10). Thus, the integral expression in Eq. (A8) with the leading-order error terms of the numerical scheme will give a more accurate continuum approximation of the discrete model.

To find the error of the numerical scheme, we present the following theorem, which is proved in Ref. 47 using the theorems in Ref. 56.

Theorem (Ref. 47). Suppose that interval $\left[b_{1}, b_{2}\right]$ is divided into $m$ subintervals with $\Delta x=\left(b_{2}-b_{1}\right) / m, x_{j}=b_{1}+(j$ -1) $\Delta x, \quad j=1, \ldots, m+1$. Let $G(x)=g_{1}(x) \log |x-t|+g_{2}(x) /(x$ $-t)+g_{3}(x)$ with $t=x_{j_{0}}$ for some $j_{0}$, where $g_{1}(x), g_{2}(x)$, and $g_{3}(x)$ are twice continuously differentiable functions. Then

$$
\begin{aligned}
\int_{b_{1}}^{b_{2}} G(x) d x= & \Delta x\left[\frac{G\left(b_{1}\right)+G\left(b_{2}\right)}{2}+\sum_{2 \leq j \leq m, j \neq j_{0}} G\left(x_{j}\right)\right] \\
& +\int_{t-\Delta x / 2}^{t+\Delta x / 2} G(x) d x-(\log \pi-1) g_{1}(t) \Delta x \\
& +O\left(\Delta x^{2}\right) .
\end{aligned}
$$

Denote

$$
G(h)=\frac{K(m)}{r(h)+r\left(h_{n}\right)}+\frac{E(m)}{r(h)-r\left(h_{n}\right)}
$$

to be the integrand in the integral expression $\mu_{m}^{0}\left(h_{n}\right)$ in Eq. (A10), with

$$
m=\frac{4 r\left(h_{n}\right) r(h)}{\left[r\left(h_{n}\right)+r(h)\right]^{2}} .
$$

Using Taylor expansions for the expressions in Eqs. (A12) and (A13) at $h=h_{n}$, and the following expansions which can be obtained from the properties of $K(m)$ and $E(m)$ as $m$ $\rightarrow 1$ (Refs. 57 and 58) $\left(m \rightarrow 1\right.$ when $\left.h \rightarrow h_{n}\right)$ :

$$
\begin{gathered}
K(m)=\log \frac{4}{(1-m)^{1 / 2}}+\frac{1}{4}\left[\log \frac{4}{(1-m)^{1 / 2}}-1\right](1-m) \\
+O\left((1-m)^{2} \log (1-m)\right), \\
E(m)=1+\frac{1}{2}\left[\log \frac{4}{(1-m)^{1 / 2}}-\frac{1}{2}\right](1-m) \\
+O\left((1-m)^{2} \log (1-m)\right),
\end{gathered}
$$

we have 


$$
\begin{aligned}
G(h)= & \frac{1}{r_{n}^{\prime}}\left(h-h_{n}\right)^{-1}-\frac{r_{n}^{\prime \prime}}{2\left(r_{n}^{\prime}\right)^{2}}+\frac{1}{2 r_{n}} \log \frac{8 r_{n}}{\left|r_{n}^{\prime}\right|\left|h-h_{n}\right|}-\frac{r_{n}^{\prime}}{8 r_{n}^{2}} \\
& \times\left(h-h_{n}\right) \log \frac{8 r_{n}}{\left|r_{n}^{\prime}\right|\left|h-h_{n}\right|} \\
& -\frac{8 r_{n}^{2} r_{n}^{\prime \prime \prime}-9\left(r_{n}^{\prime}\right)^{3}+12 r_{n} r_{n}^{\prime} r_{n}^{\prime \prime}}{48\left(r_{n} r_{n}^{\prime}\right)^{2}}\left(h-h_{n}\right)+\frac{\left(r_{n}^{\prime}\right)^{2}-2 r_{n} r_{n}^{\prime \prime}}{32 r_{n}^{3}} \\
& \times\left(h-h_{n}\right)^{2} \log \frac{8 r_{n}}{\left|r_{n}^{\prime}\right|\left|h-h_{n}\right|}+O\left(\left(h-h_{n}\right)^{2}\right),
\end{aligned}
$$

where $r_{n}^{\prime}=r^{\prime}\left(h_{n}\right)$ and $r_{n}^{\prime \prime}=r^{\prime \prime}\left(h_{n}\right)$.

It can be seen that $G(h)$ satisfies the conditions in the above theorem. Applying the above theorem to the integral in Eq. (A10) with $\Delta h=a$, we have

$$
\begin{aligned}
\mu_{m}^{0}\left(r_{n}\right)= & -\frac{(1-\nu) \sigma_{0}^{2}}{\pi G}\left\{a \left\{\frac{G(0)+G(H)}{2}+\sum_{j \neq n}\left[\frac{K\left(m_{j}\right)}{r_{j}+r_{n}}\right.\right.\right. \\
& \left.\left.+\frac{E\left(m_{j}\right)}{r_{j}-r_{n}}\right]\right\}+\int_{h_{n}-(1 / 2) a}^{h_{n}+(1 / 2) a} G(h) d h-(\log \pi-1) \\
& \left.\times\left(-\frac{1}{2 r_{n}}\right) a+O\left(a^{2}\right)\right\} .
\end{aligned}
$$

Then using Eq. (A7), we have the approximation

$$
\begin{aligned}
\tilde{\mu}_{m}\left(r_{n}\right)= & \mu_{m}^{0}\left(r_{n}\right)-\frac{(1-\nu) \sigma_{0}^{2} a}{\pi G} \int_{-\infty}^{\infty} \delta(\omega)\left[\frac{K\left(m_{\omega}\right)}{\omega+2 r_{n}}\right. \\
& \left.+\frac{E\left(m_{\omega}\right)}{\omega}\right] d \omega+\frac{(1-\nu) \sigma_{0}^{2}}{\pi G}\left\{a \frac{G(0)+G(H)}{2}\right. \\
& \left.+\int_{h_{n}-(1 / 2) a}^{h_{n}+(1 / 2) a} G(h) d h+\frac{1}{2 r_{n}}(\log \pi-1) a+O\left(a^{2}\right)\right\} .
\end{aligned}
$$

Now we simplify the right-hand side of the above equation. First, we consider the integral involving delta function, which is $\widetilde{\mu}_{m}^{\text {self }}\left(r_{n}\right)$ given by Eq. (A2). By Taylor expansions at $\omega=0$ and using Eqs. (A14) and (A15), we have

$$
\begin{aligned}
\frac{K\left(m_{\omega}\right)}{\omega+2 r_{n}}+\frac{E\left(m_{\omega}\right)}{\omega}= & \omega^{-1}+\frac{1}{2 r_{n}} \log \frac{8 r_{n}}{|\omega|}-\frac{1}{8 r_{n}^{2}} \omega \log \frac{8 r_{n}}{|\omega|}+\frac{3}{16 r_{n}^{2}} \omega \\
& +\frac{5}{32 r_{n}^{3}} \omega^{2} \log \frac{8 r_{n}}{|\omega|}+O\left(\omega^{2}\right) .
\end{aligned}
$$

Thus we have

$$
\int_{-\infty}^{\infty} \delta(\omega)\left[\frac{K\left(m_{\omega}\right)}{\omega+2 r_{n}}+\frac{E\left(m_{\omega}\right)}{\omega}\right] d \omega=\frac{1}{2 r_{n}} \log \frac{8 r_{n}}{r_{c}}+O\left(a^{3} \log a\right),
$$

where $r_{c}$ is defined by $\log r_{c}=\int_{-\infty}^{\infty} \delta(\omega) \log |\omega| d \omega$.

Next, we consider the integral of $G(h)$ over the small interval centered at $h_{n}$ on the right-hand side of Eq. (A18). Using Eq. (A16), we have

$$
\begin{aligned}
\int_{h_{n}-(1 / 2) a}^{h_{n}+(1 / 2) a} G(h) d h= & {\left[\frac{1}{2 r_{n}} \log \frac{16 r_{n}}{\left|r_{n}^{\prime}\right| a}-\frac{r_{n}^{\prime \prime}}{2\left(r_{n}^{\prime}\right)^{2}}+\frac{1}{2 r_{n}}\right] a } \\
& +O\left(a^{3} \log a\right) .
\end{aligned}
$$

Finally, we consider the terms $G(0)$ and $G(H)$ on the right-hand side of Eq. (A18). We neglect the effect of the boundary by assuming that $r_{n}>0$ and $H \rightarrow \infty$. Thus when $h$ $\rightarrow 0, r(h) \rightarrow \infty, m=\frac{4 r_{n} r(h)}{\left[r_{n}+r(h)\right]^{2}} \rightarrow 0, K(m) \rightarrow K(0)=\frac{\pi}{2}$, and $E(m)$ $\rightarrow E(0)=\frac{\pi}{2}$; thus we have

$$
G(h)=\frac{K(m)}{r(h)+r_{n}}+\frac{E(m)}{r(h)-r_{n}} \rightarrow 0,
$$

as $h \rightarrow 0$. When $H \rightarrow \infty, r(H) \rightarrow 0$ and $m \rightarrow 0$; thus we have

$$
G(H) \rightarrow \frac{\pi / 2}{r_{n}}+\frac{\pi / 2}{-r_{n}}=0,
$$

as $H \rightarrow \infty$.

Summarizing Eqs. (A18)-(A22), using $r$ as the independent variable, we have

$$
\tilde{\mu}_{m}\left(r_{n}\right) \approx \mu_{m}^{0}\left(r_{n}\right)+\frac{(1-\nu) \sigma_{0}^{2} a}{2 \pi G}\left(\frac{1}{r_{n}} \log \frac{2 \pi r_{c}\left|h^{\prime}\left(r_{n}\right)\right|}{a}+\frac{h^{\prime \prime}\left(r_{n}\right)}{h^{\prime}\left(r_{n}\right)}\right) .
$$

The right-hand side of this equation is the continuum model in Eq. (23).

\footnotetext{
*maxiang@ust.hk

${ }^{1}$ A. Pimpinelli and J. Villain, Physics of Crystal Growth (Cambridge University Press, New York, 1998).

${ }^{2}$ H. Gao and W. D. Nix, Annu. Rev. Mater. Sci. 29, 173 (1999).

${ }^{3}$ L. B. Freund and S. Suresh, Thin Film Materials: Stress, Defect Formation and Surface Evolution (Cambridge University Press, New York, 2003).

${ }^{4}$ P. Müller and A. Saúl, Surf. Sci. Rep. 54, 157 (2004).

${ }^{5}$ W. W. Mullins, J. Appl. Phys. 28, 333 (1957).

${ }^{6}$ R. J. Asaro and W. A. Tiller, Metall. Trans. 3, 1789 (1972).

${ }^{7}$ M. A. Grinfeld, Sov. Phys. Dokl. 31, 831 (1986).

${ }^{8}$ D. J. Srolovitz, Acta Metall. 37, 621 (1989).
}

${ }^{9}$ B. J. Spencer, P. W. Voorhees, and S. H. Davis, Phys. Rev. Lett. 67, 3696 (1991).

${ }^{10}$ L. B. Freund and F. Jonsdottir, J. Mech. Phys. Solids 41, 1245 (1993).

${ }^{11}$ W. H. Yang and D. J. Srolovitz, Phys. Rev. Lett. 71, 1593 (1993).

${ }^{12}$ C. H. Chiu and H. Gao, Int. J. Solids Struct. 30, 2983 (1993).

${ }^{13}$ W. H. Yang and D. J. Srolovitz, J. Mech. Phys. Solids 42, 1551 (1994).

${ }^{14}$ B. J. Spencer and D. I. Meiron, Acta Metall. Mater. 42, 3629 (1994).

${ }^{15}$ Y. W. Zhang and A. F. Bower, J. Mech. Phys. Solids 47, 2273 
(1999).

${ }^{16}$ Y. Xiang and W. E, J. Appl. Phys. 91, 9414 (2002).

${ }^{17}$ W. K. Burton, N. Cabrera, and F. Frank, Philos. Trans. R. Soc. London, Ser. A 243, 299 (1951).

${ }^{18}$ V. I. Marchenko and A. Y. Parshin, Sov. Phys. JETP 52, 129 (1980).

${ }^{19}$ O. L. Alerhand, D. Vanderbilt, R. D. Meade, and J. D. Joannopoulos, Phys. Rev. Lett. 61, 1973 (1988).

${ }^{20}$ J. M. Rickman and D. J. Srolovitz, Surf. Sci. 284, 211 (1993).

${ }^{21}$ J. Stewart, O. Pohland, and J. M. Gibson, Phys. Rev. B 49, 13848 (1994).

${ }^{22}$ R. V. Kukta and K. Bhattacharya, J. Mech. Phys. Solids 50, 615 (2002).

${ }^{23}$ J. Tersoff and E. Pehlke, Phys. Rev. Lett. 68, 816 (1992).

${ }^{24}$ J. Tersoff, Y. H. Phang, Z. Zhang, and M. G. Lagally, Phys. Rev. Lett. 75, 2730 (1995).

${ }^{25}$ F. Liu, J. Tersoff, and M. G. Lagally, Phys. Rev. Lett. 80, 1268 (1998).

${ }^{26}$ C. Duport, P. Nozieres, and J. Villain, Phys. Rev. Lett. 74, 134 (1995).

${ }^{27}$ C. Duport, P. Politi, and J. Villain, J. Phys. I 5, 1317 (1995).

${ }^{28}$ B. Houchmandzadeh and C. Misbah, J. Phys. I 5, 685 (1995).

${ }^{29}$ F. Léonard and J. Tersoff, Appl. Phys. Lett. 83, 72 (2003).

${ }^{30}$ M. Ozdemir and A. Zangwill, Phys. Rev. B 42, 5013 (1990).

${ }^{31}$ V. M. Kaganer and K. H. Ploog, Phys. Rev. B 64, 205301 (2001).

${ }^{32}$ F. Lançon and J. Villain, Phys. Rev. Lett. 64, 293 (1990).

${ }^{33}$ V. B. Shenoy and L. B. Freund, J. Mech. Phys. Solids 50, 1817 (2002).

${ }^{34}$ Y. Xiang, SIAM J. Appl. Math. 63, 241 (2002).

${ }^{35}$ Y. Xiang and W. E, Phys. Rev. B 69, 035409 (2004).

${ }^{36}$ R. M. Tromp and M. C. Reuter, Phys. Rev. Lett. 68, 820 (1992).

${ }^{37}$ N. Israeli and D. Kandel, Phys. Rev. B 60, 5946 (1999).

${ }^{38}$ S. Paulin, F. Gillet, O. Pierre-Louis, and C. Misbah, Phys. Rev. Lett. 86, 5538 (2001).

${ }^{39}$ D. Margetis, M. J. Aziz, and H. A. Stone, Phys. Rev. B 69, 041404(R) (2004).

${ }^{40}$ D. Margetis, M. J. Aziz, and H. A. Stone, Phys. Rev. B 71, 165432(R) (2005).
${ }^{41}$ D. Margetis, P. W. Fok, M. J. Aziz, and H. A. Stone, Phys. Rev. Lett. 97, 096102 (2006).

${ }^{42}$ D. Margetis and R. V. Kohn, Multiscale Model. Simul. 5, 729 (2006).

${ }^{43}$ R. V. Kukta and K. Bhattacharya, Thin Solid Films 357, 35 (1999).

${ }^{44}$ V. B. Shenoy, Appl. Phys. Lett. 85, 2376 (2004).

${ }^{45}$ F. Haußer, M. E. Jabbour, and A. Voigt, Multiscale Model. Simul. 6, 158 (2007).

${ }^{46}$ A. Ramasubramaniam and V. B. Shenoy, J. Appl. Phys. 95, 7813 (2004).

${ }^{47}$ H. Xu and Y. Xiang, SIAM J. Appl. Math. 69, 1393 (2009).

${ }^{48}$ G. Ehrlich and F. Hudda, J. Chem. Phys. 44, 1039 (1966).

${ }^{49}$ R. L. Schwoebel, J. Appl. Phys. 40, 614 (1969).

${ }^{50}$ G. S. Bales and A. Zangwill, Phys. Rev. B 41, 5500 (1990).

${ }^{51}$ K. L. Johnson, Contact Mechanics (Cambridge University Press, New York, 1985).

${ }^{52}$ These values of $g_{1}$ and $g_{3}$ were used in the simulations using the 1+1-dimensional model in Ref. 33 and are consistent with the atomistic calculations for steps on surfaces near (001) of $\mathrm{Si}$ (Refs. 59-61).

${ }^{53}$ The behaviors when $l_{t}<2 \pi r_{c} / e$ are not shown in Fig. 7. When $l_{t}<2 \pi r_{c} / e$, the adjacent steps are in the core regions of each other, more accurate results can be obtained by including the atomistic information inside the core.

${ }^{54}$ J. P. Boyd, Chebyshev and Fourier Spectral Methods, 2nd ed. (Dover, Mineola, 2001).

${ }^{55}$ R. M. Tromp and M. C. Reuter, Phys. Rev. B 47, 7598 (1993).

${ }^{56}$ A. Sidi and M. Israeli, J. Sci. Comput. 3, 201 (1988).

${ }^{57}$ P. F. Byrd and M. D. Friedman, Handbook of Elliptic Integrals for Engineers and Scientists (Springer-Verlag, New York, 1971).

${ }^{58}$ E. T. Whittaker and G. N. Watson, A Course of Modern Analysis (Cambridge University Press, England, 1990).

${ }^{59}$ T. W. Poon, S. Yip, P. S. Ho, and F. F. Abraham, Phys. Rev. B 45, 3521 (1992).

${ }^{60}$ H. J. W. Zandvliet, Rev. Mod. Phys. 72, 593 (2000).

${ }^{61}$ V. B. Shenoy, C. V. Ciobanu, and L. B. Freund, Appl. Phys. Lett. 81, 364 (2002). 